\title{
Self-dual strings and 2D SYM
}

\author{
Kazuo Hosomichi ${ }^{a}$ and Sungjay Lee ${ }^{b}$ \\ ${ }^{a}$ Department of Physics, National Taiwan University, \\ No. 1, Sec. 4, Roosevelt Road, Taipei 10617, Taiwan \\ ${ }^{b}$ Enrico Fermi Institute, University of Chicago, \\ 5620 Ellis Av., Chicago, IL 60637, U.S.A.
}

E-mail: hosomiti@yukawa.kyoto-u.ac.jp, sjlee79@uchicago.edu

ABstract: We study the system of M2-branes suspended between parallel M5-branes using ABJM model with a natural half-BPS boundary condition. For small separation between M5-branes, the worldvolume theory is shown to reduce to a $2 \mathrm{D} \mathcal{N}=(4,4)$ super Yang-Mills theory with some similarity to $q$-deformed Yang-Mills theory. The gauge coupling is related to the position of the branes in an interesting manner. The theory is considerably different from the 2D theory proposed for multiple "M-strings". We make a detailed comparison of elliptic genus of the two descriptions and find only a partial agreement.

Keywords: Brane Dynamics in Gauge Theories, M-Theory

ArXiv EPRINT: 1406.1802 


\section{Contents}

1 Introduction and summary 1

2 The setup 2

3 ABJM model on a slab 4

3.1 The bulk theory 4

3.2 Boundary condition at M5-branes 5

3.3 Boundary term 8

3.4 Nahm equation 8

4 Worldsheet theory on self-dual strings $\quad 10$

$\begin{array}{ll}4.1 \text { Derivation via dimensional reduction } & 10\end{array}$

4.2 q-deformed Yang-Mills revisited 13

$\begin{array}{lll}4.3 & \text { Derivation via brane construction } & 17\end{array}$

$\begin{array}{ll}4.4 \text { Quiver models } & 18\end{array}$

5 Elliptic genus $\quad 20$

5.1 IIA brane model 23

$\begin{array}{lll}5.2 & \text { ABJM slab } & 27\end{array}$

6 Concluding remarks $\quad 32$

A $U(1) \times U(1)$ ABJM model on a slab 33

\section{Introduction and summary}

The $(2,0)$ theory in six dimensions, which describes the many-body dynamics of M5-branes, is the unique superconformal field theory with maximal supersymmetry and in the highest possible dimensions. Despite its unique character, our understanding of this theory is far from satisfactory mainly due to the lack of Lagrangian description, although many interesting discovery have been made with regard to its property under compactifications.

As one of its distinct features, the $(2,0)$ theory has tensionless self-dual strings as its fundamental degree of freedom. On the Coulomb branch of the theory where the M5-branes are placed at distances from one another, these strings are described by open M2-branes suspended between the M5-branes [1]. The BPS spectrum of the worldsheet theory of the self-dual strings have been studied in $[1,2]$ in a dual IIA Calabi-Yau background using the refined topological vertex formalism. In this paper we study the same system using the ABJM model [3]. 
In section 2 we present the brane configuration in M-theory and summarize the symmetry of the system. In section 3 we review and extend the analysis of half-BPS boundary conditions of the ABJM model [4], focusing particularly on the one provided by M5-branes. We also study the M-theoretic analogue of the Nahm equation for ground states of multiple M2-branes suspended between M5-branes. It has been known that the M2-branes develop Nahm-like pole near their endpoints at M5-branes, but we find this occurs only when the system of M2 and M5-branes is at the origin of the transverse space $\mathbb{C}^{2} / \mathbb{Z}_{k}$.

In section 4 we take the separation between M5-branes to be very small and reduce the worldvolume theory of suspended M2-branes to two dimensions. The resulting theory, which we call the ABJM slab, is generically the $\mathcal{N}=(4,4)$ super Yang-Mills theory with R-symmetry $\mathrm{SU}(2)^{3}$, with the gauge coupling $g^{2}$ determined from the distance between the brane composite and the $\mathbb{Z}_{k}$ orbifold singularity. Since it is a dimensional reduction of a Chern-Simons gauge theory, the kinetic term for the gauge field takes the first order form $\operatorname{Tr}\left(\phi F_{01}+g^{2} \phi^{2}\right)$, and the adjoint scalar $\phi$ is subject to a certain periodicity. The gauge coupling vanishes when the branes are right at the orbifold singularity, and then the theory simplifies to a topological Yang-Mills theory coupled to adjoint matters with $\mathcal{N}=(4,4)$ supersymmetry and an enhanced R-symmetry $\mathrm{SU}(2)^{4}$. There is some similarity between the theory we obtained and the so-called $q$-deformed Yang-Mills theory [5]; some detailed comparison is made in section 4.2. Also, our theory is rather different from the theory proposed in [2] based on brane construction in a dual type IIA framework. This model will be called the IIA brane model in this paper and is reviewed in sections 4.3 and 4.4.

In section 5 we study the elliptic genus, namely the $T^{2}$ partition function, for the two descriptions of multiple self-dual strings as a function of fugacity variables $\epsilon_{1}, \epsilon_{2}, m$ parametrizing twists in the periodicity of fields. It was claimed in [2] that the formula for the elliptic genus obtained from refined topological vertex [1] can be reproduced using the IIA brane model. In section 5.1 we quickly confirm this using the idea of Higgs branch localization. Then the elliptic genus of the ABJM slab is studied in section 5.2. There it is argued that the cases $g^{2}>0$ and $g^{2}=0$ require different treatment, and the former corresponds to the special choice $m= \pm \frac{1}{2}\left(\epsilon_{1}+\epsilon_{2}\right)$ of the fugacity parameters. Moreover it is shown that the elliptic genera of the IIA brane model and the ABJM slab behave differently in the limit $m \rightarrow \pm \frac{1}{2}\left(\epsilon_{1}+\epsilon_{2}\right)$. On the other hand we show, using the JeffreyKirwan residue formula [6, 7], that the elliptic genus for $g^{2}>0$ is that of $\mathcal{N}=(4,4)$ super Yang-Mills theory and is expressed as a Young diagram sum.

We conclude with a few remarks in section 6. The appendix A shows an off-shell supersymmetric formulation of ABJM model on a slab for the simplest case $N=1$.

\section{The setup}

In this paper we study the system of parallel M2-branes suspended between parallel M5branes using the ABJM model [3].

The coordinates $\left(x_{0}, x_{1}, x_{2}\right)$ are used for the M2-brane worldvolume direction, and the transverse direction is parametrized either by eight real coordinates $\left(x_{3}, \cdots, x_{9}, x_{\natural}\right)$ or four complex coordinates $\left(z_{1}, \cdots, z_{4}\right) \equiv\left(x_{3}+i x_{4}, \cdots, x_{9}+i x_{\natural}\right)$. The ABJM model describes the 
transverse geometry $\mathbb{C}^{4} / \mathbb{Z}_{k}$, where the $\mathbb{Z}_{k}$ orbifold rotates the phase of $z_{i}$ simultaneously. In addition, we introduce M5-branes with the worldvolume direction (013456), mutually separated in the $x_{2}$ direction. We mostly focus on the case with two M5-branes, one at $x_{2}=0$ and the other at $x_{2}=L$, with $N$ M2-branes suspended between them. They are also labeled by the common position in the remaining 4 transverse direction (789দ). Our brane configuration is summarized in the table below.

\begin{tabular}{|l|ccccccccccc|}
\hline & 0 & 1 & 2 & 3 & 4 & 5 & 6 & 7 & 8 & 9 & $\emptyset$ \\
\hline M2 & - & - & $\dashv$ & & & & & & & & \\
\hline M5 & - & - & & - & - & - & - & & & & \\
\hline
\end{tabular}

Note that, if the branes are at $x_{7}=\cdots=x_{\natural}=0$, each M5-brane has the worldvolume $\mathbb{R}^{1,1}(012) \times \mathbb{R}^{4} / \mathbb{Z}_{k}(3456)$. Otherwise their worldvolume is $\mathbb{R}^{1,1} \times \mathbb{R}^{4}$.

Let us study how many supersymmetry is preserved by this system, by using the set of 11D Gamma matrices $\Gamma^{a}$. In our convention $\Gamma^{0}$ is anti-Hermitian and the rest are all Hermitian, and they satisfy

$$
\Gamma^{0} \Gamma^{1} \cdots \Gamma^{9} \Gamma^{\natural}=\mathbf{1}_{32 \times 32}
$$

We denote by $Q$ the 32-component supersymmetry on 11D flat spacetime.

First, the M2-branes (012) are half-BPS objects preserving the supersymmetry characterized by

$$
Q=\Gamma^{012} Q=\Gamma^{3456789 \natural} Q .
$$

The $\mathbb{Z}_{k}$ orbifolding for $k \geq 3$ reduces further the unbroken supersymmetry to those satisfying

$$
\exp \left\{\frac{\pi}{k}\left(\Gamma^{34}+\Gamma^{56}+\Gamma^{78}+\Gamma^{9 \natural}\right)\right\} Q=Q .
$$

Working with the basis of $11 \mathrm{D}$ spinors diagonalizing $\left(i \Gamma^{34}, i \Gamma^{56}, i \Gamma^{78}, i \Gamma^{9 \natural}\right)$, this projects out the $2 / 8$ of the unbroken supersymmetry characterized by the eigenvalues $(++++)$ and $(----)$. One is thus left with 12 supercharges or $3 \mathrm{D} \mathcal{N}=6$ supersymmetry on the M2-brane worldvolume in the absence of the M5-branes.

The M5-branes (013456) further reduce the unbroken supersymmetry to those satisfying

$$
Q=\Gamma^{013456} Q .
$$

They also introduce the boundary to the M2-brane worldvolume. The remaining supercharges are the following six, characterized by the eigenvalues

$$
\begin{aligned}
& \left(\Gamma^{01}, \Gamma^{2}, i \Gamma^{34}, i \Gamma^{56}, i \Gamma^{78}, i \Gamma^{9 \natural}\right) \\
& =(+++-+-),(+++--+),(++-++-),(++-+-+), \\
& \quad(--++--),(----++) .
\end{aligned}
$$

The M2-branes suspended between the M5-branes have the worldvolume $\mathbb{R}^{1,1} \times$ (interval), and are described at low energy by a $2 \mathrm{D}$ theory. If one identifies $\Gamma^{01}$ with the chirality of $2 \mathrm{D}$ spinors, the resulting theory is expected to have 4 chiral and 2 anti-chiral 2D supercharges. 
The M5-branes also break the $\mathrm{SU}(4) \mathrm{R}$-symmetry of the 3D $\mathcal{N}=6$ supersymmetric theory to $\mathrm{SU}(2) \times \mathrm{SU}(2) \times \mathrm{U}(1)$. The latter is in agreement with the R-symmetry of the $2 \mathrm{D}$ $\mathcal{N}=(4,2)$ supersymmetric systems.

We note here that one can introduce M5-branes (01789দ) or 9-branes (013456789দ) of appropriate orientation without breaking supersymmetry further.

\section{$3 \quad$ ABJM model on a slab}

The system of parallel M2-branes probing the transverse geometry $\mathbb{C}^{4} / \mathbb{Z}_{k}$ is described by the ABJM model [3]. To describe M2-branes suspended between M5-branes, we consider the model on a slab $\mathbb{R}^{1,1} \times($ interval) with suitable boundary conditions. We summarize here the construction of the model.

We work in the 3D Minkowski spacetime with $(-++)$ signature. Spinors are 2component quantities on which $2 \times 2$ gamma matrices act as

$$
\gamma_{m} \psi \equiv\left(\gamma_{m}\right)_{\alpha}^{\beta} \psi_{\beta} . \quad(\alpha, \beta= \pm)
$$

Bilinear products of spinors are defined using the real anti-symmetric matrix $C^{\alpha \beta}$ as follows.

$$
\xi \psi \equiv C^{\alpha \beta} \xi_{\alpha} \psi_{\beta}, \quad \xi \gamma_{m} \psi \equiv\left(C \gamma_{m}\right)^{\alpha \beta} \xi_{\alpha} \psi_{\beta} .
$$

We use

$$
C^{\alpha \beta}=\left(\begin{array}{cc}
0 & 1 \\
-1 & 0
\end{array}\right), \quad \gamma^{0}=\left(\begin{array}{cc}
0 & 1 \\
-1 & 0
\end{array}\right), \quad \gamma^{1}=\left(\begin{array}{ll}
0 & 1 \\
1 & 0
\end{array}\right), \quad \gamma^{2}=\left(\begin{array}{cc}
1 & 0 \\
0 & -1
\end{array}\right),
$$

so that $\left(C \gamma^{m}\right)^{\alpha \beta}$ are all real symmetric and moreover $\gamma^{0} \gamma^{1} \gamma^{2}=1$. Also, the components $\psi_{+}, \psi_{-}$of a spinor $\psi$ are for the eigenvalues of the chirality $\gamma^{2}=\gamma^{01}= \pm 1$ upon dimensional reduction to $2 \mathrm{D}$.

\subsection{The bulk theory}

The ABJM model is a $3 \mathrm{D} \mathcal{N}=6$ supersymmetric $\mathrm{U}(N)_{k} \times \mathrm{U}(N)_{-k}$ Chern-Simons theory coupled to bifundamental scalars $Z_{a}$ and spinors $\Psi^{a}$, where $a=1, \cdots, 4$ is the $\mathrm{SU}(4) \mathrm{R}$ symmetry index. All the fields are $N \times N$ matrix-valued, and the Lagrangian takes the form

$$
\mathcal{L}=\mathcal{L}_{\mathrm{CS}}+\mathcal{L}_{\text {kin }}+\mathcal{L}_{\text {yuk }}+\mathcal{L}_{\text {pot }},
$$

with

$$
\begin{aligned}
\mathcal{L}_{\mathrm{CS}}= & \frac{k}{4 \pi} \epsilon^{m n p} \operatorname{Tr}\left(A_{m} \partial_{n} A_{p}-\frac{2 i}{3} A_{m} A_{n} A_{p}-\tilde{A}_{m} \partial_{n} \tilde{A}_{p}+\frac{2 i}{3} \tilde{A}_{m} \tilde{A}_{n} \tilde{A}_{p}\right), \\
\mathcal{L}_{\text {kin }}= & \operatorname{Tr}\left(i \bar{\Psi}_{a} \gamma^{m} D_{m} \Psi^{a}-D_{m} \bar{Z}^{a} D^{m} Z_{a}\right), \\
\mathcal{L}_{\text {yuk }}= & \frac{2 \pi i}{k} \operatorname{Tr}\left(\Psi^{a} \bar{\Psi}_{a} Z_{b} \bar{Z}^{b}-\bar{\Psi}_{a} \Psi^{a} \bar{Z}^{b} Z_{b}-2 Z_{a} \bar{Z}^{b} \Psi^{a} \bar{\Psi}_{b}+2 \bar{Z}^{a} Z_{b} \bar{\Psi}_{a} \Psi^{b}\right. \\
& \left.\quad+\epsilon^{a b c d} Z_{a} \bar{\Psi}_{b} Z_{c} \bar{\Psi}_{d}-\epsilon_{a b c d} \bar{Z}^{a} \Psi^{b} \bar{Z}^{c} \Psi^{d}\right) \\
\mathcal{L}_{\text {pot }}= & \frac{4 \pi^{2}}{3 k^{2}} \operatorname{Tr}\left(4 Z_{a} \bar{Z}^{b} Z_{c} \bar{Z}^{a} Z_{b} \bar{Z}^{c}-6 Z_{a} \bar{Z}^{b} Z_{b} \bar{Z}^{a} Z_{c} \bar{Z}^{c}\right. \\
& \left.\quad+Z_{a} \bar{Z}^{a} Z_{b} \bar{Z}^{b} Z_{c} \bar{Z}^{c}+Z_{a} \bar{Z}^{b} Z_{b} \bar{Z}^{c} Z_{c} \bar{Z}^{a}\right) .
\end{aligned}
$$


In our convention the covariant derivative acting on bifundamental fields is defined by, for example, $D Z_{a} \equiv \mathrm{d} Z_{a}-i A Z_{a}+i Z_{a} \tilde{A}$. Therefore the gauge field strength is defined by $F=\mathrm{d} A-i A^{2}$ and similarly for $\tilde{F}$.

The $\mathcal{N}=6$ supersymmetry is parametrized by a spinor $\xi_{a b}$ satisfying $\xi_{a b}=-\xi_{b a}$ and the reality condition $\left(\xi_{a b}\right)^{\dagger} \equiv \xi^{a b}=\frac{1}{2} \epsilon^{a b c d} \xi_{c d}$. The transformation rule reads

$$
\begin{aligned}
\delta Z_{a} & =i \xi_{a b} \Psi^{b} \\
\delta \bar{Z}^{a} & =i \xi^{a b} \bar{\Psi}_{b} \\
\delta \Psi^{a} & =\gamma^{m} \xi^{a b} D_{m} Z_{b}+\xi^{b c} W_{b c}^{a}, \\
\delta \bar{\Psi}_{a} & =\gamma^{m} \xi_{a b} D_{m} \bar{Z}^{b}+\xi_{b c} \bar{W}_{a}^{b c} \\
\delta A_{m} & =-\frac{2 \pi}{k}\left(\xi_{a b} \gamma_{m} \Psi^{a} \bar{Z}^{b}+Z_{a} \xi^{a b} \gamma_{m} \bar{\Psi}_{b}\right), \\
\delta \tilde{A}_{m} & =+\frac{2 \pi}{k}\left(\xi^{a b} \gamma_{m} \bar{\Psi}_{a} Z_{b}+\bar{Z}^{a} \xi_{a b} \gamma_{m} \Psi^{b}\right),
\end{aligned}
$$

where

$$
\begin{aligned}
& W_{b c}^{a}=+\frac{\pi}{k}\left\{2 Z_{b} \bar{Z}^{a} Z_{c}+\delta_{b}^{a}\left(Z_{c} \bar{Z}^{d} Z_{d}-Z_{d} \bar{Z}^{d} Z_{c}\right)\right\}-(b \leftrightarrow c), \\
& \bar{W}_{a}^{b c}=-\frac{\pi}{k}\left\{2 \bar{Z}^{b} Z_{a} \bar{Z}^{c}+\delta_{a}^{b}\left(\bar{Z}^{c} Z_{d} \bar{Z}^{d}-\bar{Z}^{d} Z_{d} \bar{Z}^{c}\right)\right\}-(b \leftrightarrow c) .
\end{aligned}
$$

Note that the scalar potential can be expressed as $-\mathcal{L}_{\text {pot }}=\frac{2}{3} \operatorname{Tr}\left(W_{b c}^{a} \bar{W}_{a}^{b c}\right)$, so it is manifestly positive definite.

\subsection{Boundary condition at M5-branes}

To describe the M2-branes suspended between two M5-branes (013456) at $x_{2}=0$ and $x_{2}=L$, we need to consider the ABJM model with suitable boundary conditions on fields. The correct boundary condition should preserve 6 of the 12 supercharges $Q_{a b \alpha}$ and the $\mathrm{SU}(2) \times \mathrm{SU}(2) \times \mathrm{U}(1)$ subgroup of the $\mathrm{SU}(4)$ R-symmetry group. The four complex scalars $Z_{a}$ are divided into two pairs by the M5-brane boundary condition. Namely,

$$
\begin{array}{ll}
Z_{I}(I=1,2): & \text { longitudinal to the M5-branes, } \\
Z_{A}(A=3,4): & \text { transverse to the M5-branes. }
\end{array}
$$

These two pairs are assigned opposite U(1) R-charges, and are transformed by the two respective $\mathrm{SU}(2)$ factors. In short, $\mathbf{4}$ of $\mathrm{SU}(4)$ decomposes into $(\mathbf{2}, \mathbf{1})_{\mathbf{1}} \oplus(\mathbf{1}, \mathbf{2})_{-\mathbf{1}}$ of the reduced R-symmetry $\mathrm{SU}(2) \times \mathrm{SU}(2) \times \mathrm{U}(1)$.

From the discussion in the previous section, the unbroken supercharges should form a $2 \mathrm{D} \mathcal{N}=(4,2)$ superalgebra. Therefore the unbroken supercharges are

$$
Q_{13+}, Q_{14+}, Q_{23+}, Q_{24+}, Q_{12-}, Q_{34-}
$$

The four chiral supercharges furnish a 4 of $\mathrm{SO}(4)$, while the two anti-chiral supercharges are $\mathrm{SO}(4)$ singlet but carry nonzero U(1) charges. In order for the supercharge $Q_{a b \alpha}$ to be 
preserved, the normal component of the corresponding supercurrent has to vanish, namely $\mathcal{J}_{a b \alpha}^{2}=0$. Therefore we put

$$
\gamma^{2} \mathcal{J}_{a b}^{2}=P_{a}^{c} P_{b}{ }^{d} \mathcal{J}_{c d}^{2}, \quad P=\operatorname{diag}(+1,+1,-1,-1)
$$

Using the doublet indices $I, J, \cdots$ and $A, B, \cdots$ for the two $\mathrm{SU}(2)$ R-symmetry, this can be rewritten as follows,

$$
\mathcal{J}_{I A+}^{2}=\mathcal{J}_{I J-}^{2}=\mathcal{J}_{A B-}^{2}=0 .
$$

The supercurrent of the ABJM model can be obtained in the standard manner. Using a spinor $\xi^{a b}=-\xi^{b a}$ satisfying also $\xi^{a b}=\frac{1}{2} \epsilon^{a b c d} \xi_{c d}$, it can be expressed as

$$
\begin{aligned}
\xi^{a b} \mathcal{J}_{a b}^{m}= & \xi^{a b} \operatorname{Tr}\left(\gamma^{n} \gamma^{m} \bar{\Psi}_{a} D_{n} Z_{b}-\gamma^{m} \bar{\Psi}_{c} W_{a b}^{c}\right) \\
& +\xi_{a b} \operatorname{Tr}\left(\gamma^{n} \gamma^{m} \Psi^{a} D_{n} \bar{Z}^{b}-\gamma^{m} \Psi^{c} \bar{W}_{c}^{a b}\right) .
\end{aligned}
$$

The boundary condition on supercurrents (3.11) must follow from that on the gauge and matter fields. Let us first find out the boundary condition on the fermions $\Psi^{a}, \bar{\Psi}_{a}$. It has to be given by an $\mathrm{SU}(2) \times \mathrm{SU}(2) \times \mathrm{U}(1)$ invariant linear equation in the fermions. Moreover, the left-right asymmetry of the unbroken supercharges suggests that the equation should involve $\gamma^{2}$. Thus the only possible form one can think of is

$$
\gamma^{2} \Psi^{a}= \pm \Psi^{b} P_{b}^{a}, \quad \gamma^{2} \bar{\Psi}_{a}= \pm P_{a}^{b} \bar{\Psi}_{b}
$$

It turns out that the boundary condition corresponding to the M5-brane (013456) corresponds to the choice of - sign. For this choice, the above boundary condition can be rewritten more explicitly as follows.

$$
\Psi_{+}^{I}=\Psi_{-}^{A}=0, \quad \bar{\Psi}_{I+}=\bar{\Psi}_{A-}=0 .
$$

The other choice of sign corresponds to the M5-brane (01789দ). These boundary conditions, along with some other half-BPS boundary conditions in the ABJM model, were studied in some detail in [4].

By combining (3.11) with (3.14), one obtains the boundary condition on bosons. The normal components of supercurrent in (3.11) are linear in the fermions, and we require the coefficients of $\Psi_{+}^{A}, \Psi_{-}^{I}, \bar{\Psi}_{A+}, \bar{\Psi}_{I-}$ to vanish on the boundary. This leads to the following conditions [4]

$$
\begin{aligned}
D_{\mu} Z_{A} & =0 \\
D_{y} Z_{I} & =\frac{2 \pi}{k}\left(Z_{I} \bar{Z}^{J} Z_{J}-Z_{J} \bar{Z}^{J} Z_{I}\right), \\
Z_{A} \bar{Z}^{I} Z_{B} & =Z_{B} \bar{Z}^{I} Z_{A}, \quad Z_{I} \bar{Z}^{A} Z_{B}=Z_{B} \bar{Z}^{A} Z_{I},
\end{aligned}
$$

and their Hermite conjugates. Here the index $\mu$ takes 0 and 1 , and we denoted the coordinate $x_{2}$ by $y$ for convenience. The first is the Dirichlet boundary condition for $Z_{A}=\left(Z_{3}, Z_{4}\right)$. They are therefore constant along the boundary, and their boundary value determines the position of the M5-brane (013456). The second is the M-theoretic analogue 
of Nahm pole [8]. The third condition determines how the shape of Nahm pole is restricted by the nonzero values of $Z_{A}$. For example, assume $Z_{A}$ takes the form

$$
Z_{A}=\left(\begin{array}{llll}
u_{A}^{(1)} \cdot \mathbf{1}_{n_{1} \times n_{1}} & & & \\
& u_{A}^{(2)} \cdot \mathbf{1}_{n_{2} \times n_{2}} & \\
& & & \ddots
\end{array}\right) .
$$

Then $Z_{I}=\left(Z_{1}, Z_{2}\right)$ can take nonzero values only within the diagonal blocks of size $n_{1} \times$ $n_{1}, n_{2} \times n_{2}$ and so on. This corresponds to the situation with several M5-branes at different points in $\mathbb{C}^{2} / \mathbb{Z}_{k}$. The $i$-th M5-brane is at $z_{A}=u_{A}^{(i)}$ and there are $n_{i}$ M2-branes ending on it.

The boundary conditions on gauge fields follow from the Dirichlet boundary condition on $Z_{A}$. By differentiating the first equation in (3.15) one more time one finds

$$
F_{\mu \nu} Z_{A}=Z_{A} \tilde{F}_{\mu \nu}
$$

which glues the two $\mathrm{U}(N)$ gauge fields. For example, if $Z_{A}$ takes the block diagonal form (3.16) along the boundary, the gauge fields can take nonzero values only within the diagonal blocks, and moreover $A_{\mu}=\tilde{A}_{\mu}$ for each block. The gauge symmetry $\mathrm{U}(N) \times \mathrm{U}(N)$ is thus broken to $\mathrm{U}\left(n_{1}\right)_{\text {diag }} \times \mathrm{U}\left(n_{2}\right)_{\operatorname{diag}} \times \cdots$ on the boundary. Note that this boundary condition on gauge fields also resolves the problem of gauge non-invariance of Chern-Simons theory with boundary. Namely the ABJM action is gauge invariant only up to the surface term,

$$
\delta S=\frac{k}{4 \pi} \int_{\text {boundary }} \operatorname{Tr}(\alpha \mathrm{d} A-\tilde{\alpha} \mathrm{d} \tilde{A})
$$

but it vanishes if $A=\tilde{A}, \alpha=\tilde{\alpha}$ are imposed on the boundary.

More general solution to (3.17) would be that the gauge fields $A_{\mu}$ and $\tilde{A}_{\mu}$ are unitary equivalent. Namely, one could generalize (3.16) so that the $i$-th diagonal block of $Z_{A}$ is $u_{A}^{(i)}$ times a unitary matrix $U^{(i)} \in \mathrm{SU}\left(n_{i}\right)$, and relate the corresponding blocks of the gauge fields by $A_{\mu}^{(i)} U^{(i)}=U^{(i)} \tilde{A}_{\mu}^{(i)}$. One can regard the $U^{(i)}$ 's as gauge redundancy and choose them to be all identity. The $\mathrm{U}(1)$ part of the gauge group $\mathrm{U}\left(n_{i}\right)$ rotates the phase of $u_{A}^{(i)}$, but this $\mathrm{U}(1)$ rotation for general angle is not a symmetry of the ABJM model due to monopoles. As a consequence, the parameter $u_{A}^{(i)}$ is subject to the identification only under the $\mathbb{Z}_{k}$ rotation, not the full $\mathrm{U}(1)$ rotation.

The supersymmetry preserved by the boundary condition is expressed as $\xi^{a b} Q_{a b}$ with

$$
\gamma^{2} \xi_{a b}=P_{a}^{c} P_{b}{ }^{d} \xi_{c d}
$$

More explicitly, the unbroken supersymmetry is parametrized by $\xi_{+}^{I J}, \xi_{+}^{A B}$ and $\xi_{-}^{I A}$. It is straightforward to check that the boundary conditions on bosons and fermions transform among themselves under the unbroken supersymmetry. The only additional condition is that, if $Z_{A}$ takes the block-diagonal form (3.16), then the boundary value of all the fields are restricted to take the same block-diagonal form. 
Before closing this subsection, let us comment on the important special case $Z_{A}=0$. The condition (3.17) is then trivially satisfied, and one does not need to identify the two $\mathrm{U}(N)$ gauge fields along the boundary. However, if one is to relax the condition $A_{\mu}=\tilde{A}_{\mu}$ along the boundary, then the gauge invariance has to be ensured by some other means, for example by introducing boundary chiral fermions (or WZW models as was proposed in [9]). Namely, by coupling the boundary fermions of one chirality to $A_{\mu}$ and the other to $\tilde{A}_{\mu}$, one gets a desired gauge anomaly which cancels (3.18). It is not clear to us whether the $2 \mathrm{D} \mathcal{N}=(4,2)$ supersymmetry allows multiplets containing such chiral fermions. In this paper we stick to the gluing condition $A_{\mu}=\tilde{A}_{\mu}$ even when $Z_{A}=0$, and leave the study of possible boundary degree of freedom as a future problem.

\subsection{Boundary term}

We also need to check that the variational problem is well defined for the ABJM model with boundary, namely solving the bulk Euler-Lagrange equation and the boundary condition should always lead to stationary phase configurations. By a careful study of this requirement one finds that the bulk ABJM action has to be supplemented with a suitable boundary term.

The variation of the action of the ABJM model on a slab $0 \leq y \leq L$ with Lagrangian (3.4) takes the form

$$
\delta S=\int \mathrm{d}^{3} x(\text { Euler-Lagrange eqn })-\int_{y=L} \mathrm{~d}^{2} x I_{\mathrm{bd}}+\int_{y=0} \mathrm{~d}^{2} x I_{\mathrm{bd}},
$$

where the surface term $I_{\mathrm{bd}}$ reads

$$
I_{\mathrm{bd}}=\operatorname{Tr}\left(-\frac{k}{4 \pi} \varepsilon^{\mu \nu}\left(\delta A_{\mu} A_{\nu}-\delta \tilde{A}_{\mu} \tilde{A}_{\nu}\right)-i \bar{\Psi}_{a} \gamma^{2} \delta \Psi^{a}+\delta \bar{Z}^{a} D_{y} Z_{a}+D_{y} \bar{Z}^{a} \delta Z_{a}\right) .
$$

Most of the terms in $I_{\mathrm{bd}}$ vanish once the boundary conditions on fields are taken into account, except the terms proportional to $\delta Z_{I}, \delta \bar{Z}^{I}$. Using the Nahm pole boundary condition one finds

$$
I_{\mathrm{bd}}=\frac{2 \pi}{k} \operatorname{Tr}\left(\delta \bar{Z}^{I}\left(Z_{I} \bar{Z}^{J} Z_{J}-Z_{J} \bar{Z}^{J} Z_{I}\right)+\delta Z_{I}\left(\bar{Z}^{J} Z_{J} \bar{Z}^{I}-\bar{Z}^{I} Z_{J} \bar{Z}^{J}\right)\right)=\delta \mathcal{L}_{\mathrm{bd}} .
$$

Namely the surface term is nonvanishing but can be canceled by the boundary Lagrangian

$$
\mathcal{L}_{\mathrm{bd}}=\frac{\pi}{k} \operatorname{Tr}\left(\bar{Z}^{I} Z_{I} \bar{Z}^{J} Z_{J}-Z_{I} \bar{Z}^{I} Z_{J} \bar{Z}^{J}\right) .
$$

Thus the total action must include the boundary contribution

$$
S_{\text {tot }}=S_{\text {bulk }}+\int_{y=L} \mathrm{~d}^{2} x \mathcal{L}_{\text {bd }}-\int_{y=0} \mathrm{~d}^{2} x \mathcal{L}_{\text {bd }}
$$

\subsection{Nahm equation}

The word Nahm pole originally refers to a characteristic singular behavior of the solutions of Nahm equation at the boundary. As is well known, Nahm equation is a system of ordinary differential equations for a triplet of matrix valued functions [10]. The solutions are called 
Nahm data and are used to construct monopole configurations in Yang-Mills theory. In superstring theory, Nahm equation arises as the BPS equation on the worldvolume of D2branes ending on D4-branes [11]. An analogue of Nahm equation is known for M2-branes ending on M5-branes; see $[12,13]$ for earlier work and [14-18] for the work based on the ABJM model. Let us revisit this equation here.

We solve the BPS equation $\delta \Psi^{a}=\delta \bar{\Psi}_{a}=0$ with the constraint (3.19) on supersymmetry parameter $\xi_{a b}$. The solution should also preserve the $\mathrm{SO}(1,1) \times \mathrm{SU}(2) \times \mathrm{SU}(2) \times \mathrm{U}(1)$ subgroup of the 3D Lorentz and R-symmetries. The condition on bosonic fields is given by $D_{\mu} Z_{I}=D_{\mu} Z_{A}=0$, namely the fields depend only on $y \equiv x_{2}$, and

$$
\begin{aligned}
D_{y} Z_{I} & =\frac{2 \pi}{k}\left(Z_{I} \bar{Z}^{J} Z_{J}-Z_{J} \bar{Z}^{J} Z_{I}\right), \\
D_{y} Z_{A} & =\frac{2 \pi}{k}\left(Z_{A} \bar{Z}^{B} Z_{B}-Z_{B} \bar{Z}^{B} Z_{A}\right), \\
Z_{I} \bar{Z}^{A} Z_{J} & =Z_{J} \bar{Z}^{A} Z_{I}, \quad Z_{I} \bar{Z}^{J} Z_{A}=Z_{A} \bar{Z}^{J} Z_{I}, \\
Z_{A} \bar{Z}^{I} Z_{B} & =Z_{B} \bar{Z}^{I} Z_{A}, \quad Z_{A} \bar{Z}^{B} Z_{I}=Z_{I} \bar{Z}^{B} Z_{A} .
\end{aligned}
$$

The equations are symmetric under the exchange of the indices $I=1,2$ and $A=3,4$.

The first and the second equations are the Nahm equations for M2-branes ending on M5-branes (013456) or (01789 a). It turns out, however, that the solutions show nontrivial dependence on $y$ only in very limited cases, since the additional algebraic equations in the last two lines generically require that $Z_{I}, Z_{A}$ are all diagonal in a suitable gauge. Let us quickly show this. We take a generic linear combination of $Z_{A}$ which has no accidental zero eigenvalues or degeneration of eigenvalues when it is gauge-rotated to a diagonal form. Let us assume $Z_{3}$ is generic, and we gauge rotate it to the form (3.16) made of blocks which are proportional to identity matrices. Then it follows from $Z_{3} \bar{Z}^{3} Z_{I}=Z_{I} \bar{Z}^{3} Z_{3}$ that $Z_{I}, \bar{Z}^{I}$ are also block diagonal, and moreover their $j$-th blocks have to commute with each other as long as the $j$-th block of $Z_{3}$ is nonzero $\left(u_{3}^{(j)} \neq 0\right)$. Then the Nahm equation tells that the $j$-th block of $Z_{I}$ is independent of $y$. Similar argument holds with the role of $Z_{I}$ and $Z_{A}$ exchanged.

An exceptional case is when $Z_{A}=0$. Then, the algebraic equations are all satisfied without giving rise to any condition on $Z_{I}$, and the scalars $Z_{I}$ can depend on $y$ in a nontrivial manner. As an obvious generalization, if $Z_{A}$ takes the block-diagonal form (3.16) and one of the blocks is null, namely $u_{A}^{(j)}=0$ for some $j$, then $Z_{I}$ can show nontrivial $y$-dependence within the $j$-th diagonal block. Again, the same holds with the role of $Z_{A}$ and $Z_{I}$ exchanged.

M5-branes can be regarded as initial conditions for the Nahm equation. From the above analysis, one finds that the M5-branes behave like D4-branes, i.e. M2-branes develop nontrivial Nahm pole at their end, only when they are put on the $\mathbb{Z}_{k}$ orbifold singularity. If they are away from the singularity, they behave more like NS5-branes from the viewpoint of M2-branes ending on them. This is somewhat puzzling, since the difference in the behavior of M5-branes persists even in the case $k=1$ where the target space does not have any orbifold singularity. 


\section{Worldsheet theory on self-dual strings}

Let us now focus on the more specific situation of $N$ M2-branes suspended between a pair of M5-branes (013456), one at $y=0$ and the other at $y=L$. Their position in the $x_{7,8,9,4}$ direction is related to the boundary condition $Z_{A}=u_{A} \cdot \mathbf{1}_{N \times N}$. For sufficiently small $L$ we expect to find a $2 \mathrm{D}$ theory with $\mathcal{N}=(4,2)$ supersymmetry on the M2-brane worldvolume.

\subsection{Derivation via dimensional reduction}

We first derive the 2D theory by the standard dimensional reduction of the ABJM model. In this approach, 2D Lagrangian is simply $L$ times the 3D Lagrangian with the $y$-dependence of fields dropped. The resulting theory will be called the ABJM slab in this paper.

Let us look into each of the four terms in $\mathcal{L}(3.4)$. After the dimensional reduction, the Chern-Simons term with the gluing condition $A_{\mu}=\tilde{A}_{\mu}$ becomes

$$
\mathcal{L}_{\mathrm{CS}}=\frac{k}{2 \pi} \operatorname{Tr}\left(\sigma F_{01}\right), \quad \sigma \equiv A_{2}-\tilde{A}_{2}
$$

We note that $\sigma$ cannot be simply gauged away since, for arbitrary $L$, the trace of the path-ordered exponential

$$
\operatorname{Tr}\left[\mathrm{P} \exp \left(i \int_{0}^{L} d y A_{y}\right) \mathrm{P} \exp \left(i \int_{L}^{0} d y \tilde{A}_{y}\right)\right]
$$

gives a gauge-invariant observable thanks to the gluing condition on gauge fields. We also note that $\sigma$ is periodic, as $A_{2}=0$ and $A_{2}=X$ are related by a large gauge transformation for any $X$ such that $e^{i L X}=1 \in \mathrm{U}(N)$.

The matter kinetic term becomes

$$
\mathcal{L}_{\text {mat }}=\operatorname{Tr}\left(i \bar{\Psi}_{I} \gamma^{\mu} D_{\mu} \Psi^{I}+i \bar{\Psi}_{A} \gamma^{\mu} D_{\mu} \Psi^{A}-\bar{u}^{A} u_{A} \sigma^{2}-D_{\mu} \bar{Z}^{I} D^{\mu} Z_{I}-D_{y} \bar{Z}^{I} D_{y} Z_{I}\right)
$$

where the spinors $\Psi^{I}$ have only anti-chiral component, and $\Psi^{A}$ have only chiral component. The kinetic term for the fermions can be rewritten in a more explicit form,

$$
-i \operatorname{Tr}\left(\bar{\Psi}_{I-}\left(D_{0}+D_{1}\right) \Psi_{-}^{I}+\bar{\Psi}_{A+}\left(D_{0}-D_{1}\right) \Psi_{+}^{A}\right) .
$$

The last term in (4.3) cannot simply be dropped, since the boundary condition on $Z_{I}$ is not of simple Neumann type. The Yukawa and the potential terms become

$$
\begin{aligned}
\mathcal{L}_{\text {yuk }}= & \frac{4 \pi i}{k} \operatorname{Tr}\left(u_{A} \Psi^{A}\left[\bar{Z}^{I}, \bar{\Psi}_{I}\right]+\epsilon^{A B} u_{A} \bar{\Psi}_{B} \epsilon^{I J}\left[Z_{I}, \bar{\Psi}_{J}\right]\right. \\
& \left.-\bar{u}^{A} \bar{\Psi}_{A}\left[Z_{I}, \Psi^{I}\right]-\epsilon_{A B} \bar{u}^{A} \Psi^{B} \epsilon_{I J}\left[\bar{Z}^{I}, \Psi^{J}\right]\right), \\
\mathcal{L}_{\text {pot }}= & \frac{4 \pi^{2}}{k^{2}} \bar{u}^{A} u_{A} \operatorname{Tr}\left(\left[Z_{I}, Z_{J}\right]\left[\bar{Z}^{I}, \bar{Z}^{J}\right]-\left[Z_{I}, \bar{Z}^{J}\right]\left[Z_{J}, \bar{Z}^{I}\right]\right) \\
& -\frac{4 \pi^{2}}{k^{2}} \operatorname{Tr}\left(\left(\bar{Z}^{J} Z_{J} \bar{Z}^{I}-\bar{Z}^{I} Z_{J} \bar{Z}^{J}\right)\left(Z_{I} \bar{Z}^{K} Z_{K}-Z_{K} \bar{Z}^{K} Z_{I}\right)\right) .
\end{aligned}
$$


If one naively uses the second of the boundary condition (3.15), the last terms in $\mathcal{L}_{\text {mat }}$ and $\mathcal{L}_{\text {pot }}$ add up. However, if the boundary contribution of (3.24) is added in the form of a total $y$-derivative, the sum of the three can be rewritten into the following form

$$
\begin{aligned}
& -\operatorname{Tr}\left(D_{y} \bar{Z}^{I} D_{y} Z_{I}+\frac{4 \pi^{2}}{k^{2}}\left(\bar{Z}^{J} Z_{J} \bar{Z}^{I}-\bar{Z}^{I} Z_{J} \bar{Z}^{J}\right)\left(Z_{I} \bar{Z}^{K} Z_{K}-Z_{K} \bar{Z}^{K} Z_{I}\right)\right) \\
& +\frac{\pi}{k} \partial_{y} \operatorname{Tr}\left(\bar{Z}^{I} Z_{I} \bar{Z}^{J} Z_{J}-Z_{I} \bar{Z}^{I} Z_{J} \bar{Z}^{J}\right) \\
& =-\operatorname{Tr}\left(D_{y} \bar{Z}^{I}-\frac{2 \pi}{k}\left(\bar{Z}^{J} Z_{J} \bar{Z}^{I}-\bar{Z}^{I} Z_{J} \bar{Z}^{J}\right)\right)\left(D_{y} Z_{I}-\frac{2 \pi}{k}\left(Z_{I} \bar{Z}^{K} Z_{K}-Z_{K} \bar{Z}^{K} Z_{I}\right)\right),
\end{aligned}
$$

which vanishes under the boundary condition (3.15). It is interesting to notice here that, in our action integral (3.24) for the M2-M5 system, the first (bulk) term is actually not proportional to $L$ as is naively expected, but has an $L$-independent piece. This piece is canceled by the boundary term, and the total action integral is proportional to $L$.

With this cancellation understood, the remaining terms form a $2 \mathrm{D}$ supersymmetric system. The Lagrangian for generic nonzero $u_{A}$ is given by

$$
\begin{aligned}
\frac{1}{L} \cdot \mathcal{L}= & \operatorname{Tr}\left(\frac{k}{2 \pi} \sigma F_{01}+i \bar{\Psi}_{I} \gamma^{\mu} D_{\mu} \Psi^{I}+i \bar{\Psi}_{A} \gamma^{\mu} D_{\mu} \Psi^{A}-D_{\mu} \bar{Z}^{I} D^{\mu} Z_{I}-u^{2} \sigma^{2}\right) \\
& +\frac{4 \pi u}{k} \operatorname{Tr}\left(\Psi^{\dot{3}}\left[\bar{Z}^{I}, \bar{\Psi}_{I}\right]-\bar{\Psi}_{\dot{4}} \epsilon^{I J}\left[Z_{I}, \bar{\Psi}_{J}\right]+\bar{\Psi}_{\dot{3}}\left[Z_{I}, \Psi^{I}\right]+\Psi^{\dot{4}} \epsilon_{I J}\left[\bar{Z}^{I}, \Psi^{J}\right]\right) \\
& -\frac{4 \pi^{2} u^{2}}{k^{2}} \operatorname{Tr}\left(\left[Z_{I}, \bar{Z}^{J}\right]\left[Z_{J}, \bar{Z}^{I}\right]+\left[Z_{I}, Z_{J}\right]\left[\bar{Z}^{J}, \bar{Z}^{I}\right]\right),
\end{aligned}
$$

where

$$
\Psi^{\dot{3}}=\frac{i}{u} u_{A} \Psi^{A}, \quad \Psi^{\dot{4}}=-\frac{i}{u} \epsilon_{A B} \bar{u}^{A} \Psi^{B} . \quad\left(u \equiv \sqrt{u_{A} \bar{u}^{A}}\right)
$$

We expected that the dimensionally reduced Lagrangian preserves $\mathcal{N}=(4,2)$ supersymmetry but the nonzero $u_{A}$ breaks the $\mathrm{SU}(2) \times \mathrm{SU}(2) \times \mathrm{U}(1)$ R-symmetry down to an $\mathrm{SU}(2)$. But it turns out that the system actually has $\mathcal{N}=(4,4)$ supersymmetry, and the R-symmetry enhances to $\mathrm{SU}(2)^{3}$. Indeed if one introduces the notation

$$
\begin{aligned}
\text { scalar: } & Z^{I \dot{I}} \equiv\left(Z^{I \dot{1}}, Z^{I \dot{2}}\right) \equiv\left(\epsilon^{I J} Z_{J}, \bar{Z}^{I}\right), \\
\text { chiral spinor: } & \Psi^{\dot{I} \dot{A}} \equiv\left(\Psi^{\dot{1} \dot{A}}, \Psi^{\dot{2} \dot{A}}\right) \equiv\left(\Psi^{\dot{A}},-\epsilon^{\dot{A} \dot{B}} \bar{\Psi}_{\dot{B}}\right), \\
\text { anti-chiral spinor: } & \Psi^{I \dot{A}} \equiv\left(\Psi^{I \dot{3}}, \Psi^{I \dot{4}}\right) \equiv\left(\Psi^{I},-\epsilon^{I J} \bar{\Psi}_{J}\right),
\end{aligned}
$$

one can show that the above Lagrangian is invariant under three copies of $\mathrm{SU}(2)$ 's acting on the indices $I, \dot{I}$ and $\dot{A}$ respectively. The Lagrangian is also invariant under $\mathcal{N}=(4,4)$ supersymmetry transformation,

$$
\begin{aligned}
\delta Z^{I \dot{I}} & =\xi_{\dot{A}}^{\dot{I}} \Psi^{I \dot{A}}+\xi_{\dot{A}}^{I} \Psi^{\dot{I} \dot{A}} \\
\delta \Psi^{\dot{I} \dot{A}} & =-i \gamma^{\mu} \xi_{I}^{\dot{A}} D_{\mu} Z^{I \dot{I}}+i \xi^{\dot{I} \dot{A}} \sigma u-\frac{2 \pi u}{k} \xi_{\dot{J}}^{\dot{A}} \epsilon_{I J}\left[Z^{I \dot{I}}, Z^{J \dot{J}}\right], \\
\delta \Psi^{I \dot{A}} & =-i \gamma^{\mu} \xi_{\dot{I}}^{\dot{A}} D_{\mu} Z^{I \dot{I}}-i \xi^{I \dot{A}} \sigma u-\frac{2 \pi u}{k} \xi_{J}^{\dot{A}} \epsilon_{\dot{I} \dot{J}}\left[Z^{I \dot{I}}, Z^{J j}\right],
\end{aligned}
$$




$$
\begin{aligned}
\delta A_{\mu} & =-\frac{2 \pi u}{k}\left(\xi_{I \dot{A}} \gamma_{\mu} \Psi^{I \dot{A}}+\xi_{\dot{I} \dot{A}} \gamma_{\mu} \Psi^{\dot{I} \dot{A}}\right), \\
\delta \sigma & =-\frac{2 \pi i}{k}\left(\xi_{\dot{I} \dot{A}}\left[Z^{I \dot{I}}, \Psi_{I}^{\dot{A}}\right]-\xi_{I \dot{A}}\left[Z^{I \dot{I}}, \Psi_{\dot{I}}^{\dot{A}}\right]\right),
\end{aligned}
$$

of which the $\mathcal{N}=(4,2)$ part can be obtained from the reduction of $3 \mathrm{D}$ unbroken supersymmetry. Here the supersymmetry parameter $\xi^{\dot{I} \dot{A}}$ is chiral and $\xi^{I \dot{A}}$ is anti-chiral.

Most importantly, the Lagrangian for the ABJM slab looks like a $q$-deformed version of $\mathcal{N}=(4,4)$ super Yang-Mills theory. Indeed, if one forgets about the periodicity of $\sigma$ and naively integrates it out, we obtain the kinetic term for the gauge field

$$
\mathcal{L}=-\frac{1}{2 g^{2}} \operatorname{Tr}\left(F_{\mu \nu} F^{\mu \nu}\right)+\cdots, \quad g^{2}=\frac{16 \pi^{2} u^{2}}{k^{2} L} .
$$

A natural question arises at this point: how is the $\sigma^{2}$ term in the Lagrangian consistent with the periodicity of $\sigma$ ?

In fact, the Lagrangian including the auxiliary scalar $\sigma$ have been used frequently in the study of 2D pure Yang-Mills theory. In particular, [5] proposed the so-called $q$-deformed Yang-Mills theory by making $\sigma$ a periodic variable. The Lagrangian for the ABJM slab contains basically the same Yang-Mills term and periodic $\sigma$, but in its derivation the $\sigma^{2}$ term arose in a rather strange way through the boundary value of charged matter scalars. Therefore, the ABJM slab may well answer to the above question differently from the $q$-deformed Yang-Mils theory studied in [5]. We discuss this point in detail in the next subsection.

In the special case $u_{A}=0$, many terms in the Lagrangian disappear, and one is left with a topological gauge theory coupled to adjoint matters,

$$
\frac{1}{L} \cdot \mathcal{L}=\operatorname{Tr}\left(\frac{k}{2 \pi} \sigma F_{01}+i \bar{\Psi}_{I} \gamma^{\mu} D_{\mu} \Psi^{I}+i \bar{\Psi}_{A} \gamma^{\mu} D_{\mu} \Psi^{A}-D_{\mu} \bar{Z}^{I} D^{\mu} Z_{I}\right)
$$

The chiral spinors $\Psi^{\dot{A}}(4.8)$ become ill-defined, but one can instead introduce

$$
\Psi^{\dot{I} A} \equiv\left(\Psi^{\dot{1} A}, \Psi^{\dot{2} A}\right) \equiv\left(\Psi^{A},-\epsilon^{A B} \bar{\Psi}_{B}\right)
$$

and rewrite the Lagrangian using the scalars $Z^{I \dot{I}}$, anti-chiral spinors $\Psi^{I \dot{A}}$ defined in (4.9) and the above chiral spinors $\Psi^{\dot{I} A}$. One can then explicitly see that the R-symmetry is enhanced to $\mathrm{SU}(2)^{4}$, and the supersymmetry is generated by chiral spinors $\xi_{+}^{\dot{I} \dot{A}}$ and antichiral spinors $\xi_{-}^{I A}$.

The moduli space of vacua of the ABJM slab for generic $u>0$ is that of $\mathcal{N}=$ $(4,4) \mathrm{U}(N)$ super Yang-Mills theory, so it is the $N$-th symmetric product of $\mathbb{R}^{4}$. It is parametrized by a pair of $N \times N$ matrices $Z_{I}$ which commute with themselves and their Hermite conjugates. Since the effect of $\mathbb{Z}_{k}$ orbifold is to identify the system at different values of the coupling $u_{A}$, we do not need to care about its action on the fields. For $u=0$, on the other hand, the scalar potential vanishes and the relation between the moduli space and the position of M2-branes becomes unclear. Moreover one has to take care of the $\mathbb{Z}_{k}$ orbifold acting on the fields in this case. 


\section{2 $\quad q$-deformed Yang-Mills revisited}

Here we would like to compare some aspects of the 2D SUSY gauge theories obtained above with those of 2D pure Yang-Mills theory and its $q$-deformation, paying special attention to the periodicity of the scalar field. Let us first review some of the exact analysis of 2D Yang-Mills and 3D Chern-Simons theories.

It is a special feature of two dimensions that the Yang-Mills action depends only on the volume and not on any more detail of the metric of the surface. A nice way to see this feature is to regard it as a deformation of a topological field theory. Let $\Sigma_{h}$ be a Riemann surface of genus $h$. The Euclidean action on $\Sigma_{h}$ is

$$
S_{\mathrm{YM}}=\frac{1}{2 g^{2}} \int_{\Sigma_{h}} \operatorname{Tr}(F \wedge * F) \simeq \int_{\Sigma_{h}} \operatorname{Tr}\left(i \phi F+\frac{g^{2}}{2} \omega \phi^{2}\right) .
$$

Here $\omega$ is the normalized volume form of $\Sigma_{h}$ satisfying $\int_{\Sigma_{h}} \omega=1$. The theory at $g=0$ does not depend on the metric at all, so it is a topological field theory. Even after $g$ is turned on, the theory is invariant under area-preserving diffeomorphisms. Thanks to this property the 2D Yang-Mills theory is exactly solved; the partition function on Riemann surfaces of arbitrary genus has been worked out by various techniques, for example the idea of lattice gauge theory was applied in [19], and in [20] more detail of the theory was studied using non-abelian localization.

Let us look more closely into the theory using the approach of abelianization [21]. This approach is characterized by the gauge choice in which $\phi$ is diagonal. To be more explicit, let us denote a Cartan subalgebra of the gauge symmetry algebra by $\mathbf{t}$ and its complement by $\mathbf{k}$. We decompose various fields accordingly, as $\phi=\phi^{\mathbf{t}}+\phi^{\mathbf{k}}$ etc. Our (partial) gaugefixing condition is therefore $\phi^{\mathbf{k}}=0$. The remaining $\mathbf{t}$-gauge invariance can be fixed in any convenient way; for example the Lorentz gauge $\mathrm{d} * A^{\mathrm{t}}=0$.

The path integral of the system including ghosts was studied in [21]. The strategy is to integrate first over all the fields except $\phi^{\mathbf{t}}$, which in fact simply gives rise to a delta function and a determinant. The delta function arises from the integration of $\mathbf{t}$-valued fields, which requires $\phi^{\mathbf{t}}$ to be constant and take values on the weight lattice of the gauge group. The Gaussian integral over $\mathbf{k}$-valued fields gives rise to the determinant of $\phi$ acting on (k-components of) the ghosts $c, \bar{c}$ and the gauge fields $A$,

$$
\frac{\left.\operatorname{Det}(\phi)\right|_{\bar{c}^{\mathrm{k}}, c^{\mathrm{k}}}}{\left(\left.\operatorname{Det}(\phi)\right|_{A^{\mathrm{k}}}\right)^{1 / 2}}=\left(\prod_{\alpha>0} \alpha \cdot \phi\right)^{2-2 h} \text {. }
$$

Here the product is over all the positive roots $\alpha$. Identifying points on the weight lattice with gauge group representations, one can express the partition function on genus- $h$ surface as follows,

$$
Z_{h}=\text { const } \cdot \sum_{\lambda: \text { reps. }} d(\lambda)^{2-2 h} e^{-\frac{1}{2} g^{2} C_{2}(\lambda)},
$$

where $C_{2}(\lambda)=\lambda \cdot(\lambda+2 \rho)$ is the second Casimir, $\rho$ is the Weyl vector and $d(\lambda)$ is the dimension of the representation $\lambda$.

$$
d(\lambda) \equiv \prod_{\alpha>0} \frac{\alpha \cdot(\lambda+\rho)}{\alpha \cdot \rho} .
$$


The $q$-deformation introduces a periodicity to $\phi$ by regarding it not simply as an adjoint scalar but a component of the gauge field along a compact direction. It was introduced in [5] in the study of D4-branes in the topological string theory on a non-compact CalabiYau manifold. The target is given by two line bundles $L_{1} \oplus L_{2}$ fibred over a Riemann surface $\Sigma_{h}$, satisfying the Calabi-Yau condition

$$
\operatorname{deg}\left(L_{1}\right)+\operatorname{deg}\left(L_{2}\right)=2 h-2
$$

The D4-branes support a topological gauge theory with the Lagrangian $-\frac{1}{2 g_{s}} \operatorname{Tr} F \wedge F$. For $N$ D4-branes wrapping the four-dimensional non-compact submanifold $L_{2} \rightarrow \Sigma_{h}$, the theory becomes the $\mathrm{U}(N)$ Chern-Simons theory of level $k=2 \pi i / g_{s}$ on its boundary, which is a circle bundle over $\Sigma_{h}$ of monopole degree $p=\operatorname{deg}\left(L_{2}\right)$. Let us denote such a 3-manifold by $M_{h, p}$. It was argued in [5] and explained in full detail in [22] that the worldvolume gauge theory can be further reduced to a 2D q-deformed Yang-Mills theory on $\Sigma_{h}$.

The topological structure of $M_{h, p}$ is characterized by a 1-form $\kappa$ satisfying $\mathrm{d} \kappa=2 \pi p \omega$, with $\omega$ the normalized volume form on $\Sigma_{h}$ as before. Using the coordinate $\theta \sim \theta+2 \pi$ for the circle fiber and $x^{1}, x^{2}$ for the base $\Sigma_{h}$, it can be expressed as $\kappa=\mathrm{d} \theta+a_{i} \mathrm{~d} x^{i}$. The exterior derivative on $M_{h, p}$ can be decomposed as d $=\kappa \partial_{\theta}+\mathrm{d}_{H}$, and $\mathrm{d}_{H}=\mathrm{d} x^{i}\left(\partial_{i}-a_{i} \partial_{\theta}\right)$ can be used to define parallel transport along the base $\Sigma_{h}$. Using the decomposition of the gauge field $A_{(3)}=\phi \kappa+A$ into the base and fiber directions, the Chern-Simons action can be written as

$$
\begin{aligned}
S & =\frac{i k}{4 \pi} \int_{M_{h, p}} \operatorname{Tr}\left(A_{(3)} \mathrm{d} A_{(3)}-\frac{2 i}{3} A_{(3)}^{3}\right) \\
& =\frac{i k}{4 \pi} \int_{M_{h, p}} \operatorname{Tr}\left(2 \pi p \phi^{2} \kappa \wedge \omega+2 \phi \kappa \wedge \mathrm{d} A+\kappa \wedge\left(\partial_{\theta} A-i[\phi, A]\right) \wedge A\right) .
\end{aligned}
$$

Here the overall $i$ is because the Euclidean path integral weight is $e^{-S}$ in our convention.

All the fields are periodic function of $\theta$ and therefore can be decomposed into Fourier modes. The $n$-th Fourier modes couple to the gauge field $A+n a$ on $\Sigma_{h}$, so they are the sections of $\mathcal{O}(n p)$ on $\Sigma_{h}$ besides carrying the gauge charge. The Chern-Simons theory would reduce to the 2D Yang-Mills theory with gauge coupling $\sim p^{1 / 2}$ if one discarded all the non-zero modes. But in fact, no matter how small the radius of the fiber circle becomes, one can never simply discard the nonzero modes, as the Chern-Simons theory is a topological field theory.

For the computation of partition function, it is most convenient to work in the gauge $\phi^{\mathbf{k}}=\partial_{\theta} \phi^{\mathbf{t}}=0$. As before, the strategy is to path-integrate over all the fields except $\phi^{\mathbf{t}}$. Those fields are further divided into two groups. The first consists of the t-valued $\theta$-independent modes; integration over them yields a delta function requiring $\phi$ to take quantized constant values. The second group contains all the remaining modes; integrating over them gives rise to the familiar shift of the level by the dual Coxeter number $k \rightarrow \hat{k} \equiv$ $k+h^{\vee}$, as well as the determinant

$$
\left(\prod_{\alpha>0} \sin (\pi \alpha \cdot \phi)\right)^{2-2 h} .
$$


The determinant has the appropriate periodicity in $\phi$ thanks to all the Fourier modes having been taken into account. The delta function constraint now requires that $\hat{k} \cdot \phi^{\mathbf{t}}$ be on the weight lattice. Thus the partition function of $q$-deformed Yang-Mills theory is given by the same formula (4.16), with the dimension of the representations replaced by its $q$-analogue,

$$
d_{q}(\lambda) \equiv \prod_{\alpha>0} \frac{[\alpha \cdot(\lambda+\rho)]_{q}}{[\alpha \cdot \rho]_{q}}, \quad[x]_{q} \equiv \frac{q^{x / 2}-q^{-x / 2}}{q^{1 / 2}-q^{-1 / 2}} .
$$

The deformation parameter is $q \equiv \exp (2 \pi i / \hat{k})$.

Note that for integer $k$ the number of representations contributing to the partition function becomes finite. The 2D Yang-Mills coupling also depends on the renormalized Chern-Simons coupling as $g^{2}=i \hat{k} p$. The pure imaginary and quantized value of $g^{2}$ is essential for the periodicity of $\phi$.

Let us now turn to the model of our interest. If one forgets about the matter fields, the gauge sector is $\mathrm{U}(N) \times \mathrm{U}(N)$ Chern-Simons theory on the slab $\mathbb{R}^{1,1} \times$ (interval), with the identification of the two $\mathrm{U}(N)$ gauge fields at $y=0$ and $y=L$. This is effectively the same as the $\mathrm{U}(N)$ Chern-Simons theory on $\mathbb{R}^{1,1} \times S^{1}$, corresponding to the case $p=0$ in the previous paragraph. Therefore, in the absence of matters the theory would not reduce to 2D topological Yang-Mills theory in the small $L$ limit. Actually it is known that for $p=0$ a different 2D gauge theory, called $G / G$ gauged WZNW model with $G=\mathrm{U}(N)$, gives a precise description of the $3 \mathrm{D}$ system [23].

The term $u^{2} \sigma^{2}$ in (4.7) arises from the nonzero boundary value of matter scalar fields obeying Dirichlet boundary condition. Unlike the Chern-Simons theory on $M_{h, p}$, this term (in Euclidean action) is real, and there is no reason that its coefficient is quantized. So we need a different argument to resolve the contradiction that such a term is present in a system of periodic $\sigma$.

To make the argument simple and concrete, we limit our discussion here to the simplest example of Euclidean U(1) $\times \mathrm{U}(1)$ ABJM model on $S^{2} \times$ (interval), though it is not fully clear how the following argument extends to the more general cases. As is shown in the appendix, if the $S^{2}$ is round, one can introduce auxiliary fields to make the $2 \mathrm{D}$ off-shell supersymmetry manifest.

If one denotes the average and difference of the two $\mathrm{U}(1)$ gauge fields by $B_{m}$ and $C_{m}$, the Lagrangian becomes

$$
\mathcal{L}=\frac{i k}{4 \pi} \varepsilon^{m n p} B_{m} \partial_{n} C_{p}+D_{m} \bar{Z}^{a} D^{m} Z_{a}+\text { (fermions) }
$$

where the matter covariant derivative is defined as $D_{m} Z_{a}=\left(\partial_{m}-i C_{m}\right) Z_{a}$, etc. The Yukawa and bosonic potential terms all happen to vanish. The $B_{m}$ equation of motion requires $C_{m}$ to be flat. So the path integral reduces to that over a flat gauge field $C_{m}$ and some free matter fields coupled to it.

Among the path integration variables is a real constant field $\sigma=C_{y}$ which has periodicity $\sigma \sim \sigma+2 \pi / L$. The periodicity arises from the large gauge transformations

$$
\sigma^{\prime}=\sigma+\frac{2 \pi n}{L}, \quad Z_{a}^{\prime}=Z_{a} \exp \left(\frac{2 \pi i n y}{L}\right) . \quad(n \in \mathbb{Z})
$$


On the other hand, when deriving the dimensionally reduced Lagrangian (4.7), we assumed $Z_{A}=u_{A}=$ (constant) and neglected all the Kaluza-Klein modes, which clearly breaks the large gauge invariance. To restore the invariance, we need to take account of all the matter Kaluza-Klein modes. The scalars $Z_{I}$ and $Z_{A}$ obey Neumann and Dirichlet boundary conditions respectively, so for constant $C_{y}=\sigma$ they are naturally expanded into the Fourier modes

$$
\begin{aligned}
& Z_{I}\left(x^{\mu}, y\right)=\sum_{n \geq 0} Z_{I(n)}\left(x^{\mu}\right) \cdot e^{i \sigma y} \cos \frac{\pi n y}{L}, \\
& Z_{A}\left(x^{\mu}, y\right)=u_{A}+\sum_{n>0} Z_{A(n)}\left(x^{\mu}\right) \cdot e^{i \sigma y} \sin \frac{\pi n y}{L} .
\end{aligned}
$$

Now if the large gauge transformation (4.23) is applied to $Z_{A}$, its constant piece $u_{A}$ is transformed into an oscillating function, but the change can be absorbed by appropriate shifts of the mode variables $Z_{A(n)}$.

In order to see how the term $L u^{2} \sigma^{2}$ in the Lagrangian gets modified by the KaluzaKlein modes, we integrate out the free matter fields and see how the result depends on $\sigma$. We notice that, assuming $\sigma$ is constant, the $\sigma$-dependence in the Lagrangian and the Fourier decomposition (4.24) can be eliminated almost completely by a gauge transformation from $C_{y}=\sigma$ to $C_{y}=0$. This transformation is a symmetry of the system though not in the group of gauge equivalence. After that the only $\sigma$-dependence remains in the term $D_{y} \bar{Z}^{A} D_{y} Z_{A}$ in the Lagrangian. By substituting (4.24) into it, we obtain

$$
\int_{0}^{L} \mathrm{~d} y D_{y} \bar{Z}^{A} D_{y} Z_{A}=\frac{1}{L} \sum_{n>0}\left(\frac{n^{2} \pi^{2}}{2} \bar{Z}_{(n)}^{A} Z_{A(n)}+\bar{Z}_{A(n)} c_{A(n)}+c_{A(n)}^{*} Z_{A(n)}\right)+L u^{2} \sigma^{2}
$$

with

$$
c_{A(n)}=u_{A} \frac{L^{2} \sigma^{2} n \pi\left(e^{-i L \sigma+i n \pi}-1\right)}{L^{2} \sigma^{2}-n^{2} \pi^{2}} .
$$

The Gaussian integral over the mode variables $Z_{A(n)}, Z_{I(n)}$ and their superpartners does not yield $\sigma$-dependent determinant, but there remains a $\sigma$-dependent classical Lagrangian

$$
\mathcal{L}_{\mathrm{cl}}=L u^{2} \sigma^{2}-\sum_{n>0} \frac{2 c_{A(n)}^{*} c_{A(n)}}{L n^{2} \pi^{2}}=\frac{4 u^{2}}{L} \sin ^{2} \frac{\sigma L}{2} .
$$

This is the desired periodic function which approaches $L u^{2} \sigma^{2}$ for small $\sigma L$.

The Lagrangian (4.27) can be well approximated by the quadratic function and $\sigma$ can be integrated out if the saddle of the Gaussian $\sigma$-integration is sufficiently close to $\sigma=0$ and the width is narrow. One finds from (4.7) that the location of the saddle and the width (in terms of the variable of unit periodicity $L \sigma$ ) are

$$
L \sigma=\frac{k L}{4 \pi u^{2}} F_{01}=\frac{4 \pi}{k g^{2}} F_{01}, \quad\left\langle(L \sigma)^{2}\right\rangle=\frac{8 \pi^{2}}{k^{2} g^{2}} . \quad\left(g^{2} \equiv \frac{16 \pi^{2} u^{2}}{k^{2} L}\right)
$$

Therefore the periodicity becomes unimportant in the limit $L \rightarrow 0$.

More careful argument would take into account that the average value of $F_{01}$ may also depend on $L$. It would be reasonable to guess such an effect from the dependence of $\left\langle F_{01}\right\rangle$ 
of $2 \mathrm{D} \mathcal{N}=(4,4)$ super Yang-Mills theory on the gauge coupling $g^{2} \sim u^{2} / L$. $\left\langle F_{01}\right\rangle$ would therefore stay constant in the limit of small $L$ if we send $u \rightarrow 0$ at the same time so that $g^{2}$ is kept fixed. In view of (4.28), again we believe that in the limit $L \rightarrow 0$ with $u$ fixed the periodicity will become unimportant.

\subsection{Derivation via brane construction}

There is another candidate for the worldsheet theory of self-dual strings which was obtained via a dual type IIA brane construction [2]. We will hereafter call it the IIA brane model. This model can be compared with the ABJM slab at least for $k=1$, in which case the transverse geometry is simply $\mathbb{R}^{8}=\mathbb{R}_{(3456)}^{4} \times \mathbb{R}_{(789 \text { b) }}^{4}$. Here we briefly review the construction of the model.

Let us replace the $\mathbb{R}_{(789 \text { ) }}^{4}$ by a Taub-NUT space, that is a circle fibration over $\mathbb{R}^{3}$ such that the radius of the circle asymptotes to a constant $R$ at infinity but shrinks at the origin of $\mathbb{R}^{3}$. It becomes the flat $\mathbb{R}^{4}$ in the limit $R \rightarrow \infty$, so the replacement of the background does not affect the values of $R$-independent observables.

In the limit of small $R$ we move to the weakly coupled type IIA superstring theory with a single D6-brane (0123456), and the M2-M5 system turns into the D2-branes (012) suspended between parallel NS5-branes (013456). The $N$ D2-branes suspended between two NS5-branes give rise to the $\mathrm{U}(N) \mathcal{N}=(4,4)$ super Yang-Mills theory on the worldvolume, but the D6-brane breaks half of the supersymmetry and introduces additional fundamental matters. Although the supersymmetry of the D2-D6-NS5 system is half of the M2-M5 system, their worldvolume theory was shown to reproduce the elliptic genus of self-dual strings evaluated using refined topological vertex formalism $[1,2]$.

Let us choose the orientation of the IIA branes so that the unbroken supersymmetry is given by the (10D 32-component spinor) supercharge $Q$ satisfying

$$
Q=\Gamma^{012} Q=\Gamma^{013456} Q=-\Gamma^{0123456} Q,
$$

and corresponds to $\mathcal{N}=(0,4)$ in two dimensions. This is in order for the elliptic genus to be a holomorphic function of the modular parameter of the torus in the convention of $[6,7]$. The brane configuration is summarized in the table below.

\begin{tabular}{|c|cccccccccc|}
\hline & 0 & 1 & 2 & 3 & 4 & 5 & 6 & 7 & 8 & 9 \\
\hline D6 & - & - & - & - & - & - & - & & & \\
\hline D2 & - & - & - & & & & & & & \\
\hline NS5 & - & - & & - & - & - & - & & & \\
\hline
\end{tabular}

To understand the structure of the $2 \mathrm{D}$ theory, it is helpful to consider first the parallel D2-D6 system without NS5-branes, which gives rise to the 3D $\mathcal{N}=4 \mathrm{U}(N)$ gauge theory with one adjoint and one fundamental hypermultiplets. The vector multiplet contains a gauge field (one of its components becomes scalar upon dimensional reduction) and three scalars describing the motion of the D2-branes in the $x_{7,8,9}$ directions. The scalars in the adjoint hypermultiplet are for the motion in the $x_{3,4,5,6}$ direction, and the fundamental 


\begin{tabular}{|r|cccc|}
\hline multiplet & scalar & \multicolumn{2}{|c|}{ spinor } & vector \\
\hline vector & $Y^{A \dot{A}}$, & $\lambda_{+}^{\dot{I}}$, & $\lambda_{-}^{\dot{I} A}$, & $A_{\mu}$, \\
adjoint hyper & $Z^{I \dot{I}}$, & $\Psi_{+}^{I A}$, & $\Psi_{-}^{I \dot{A}}$, & \\
fundamental hyper & $q^{\dot{I}}$, & $\psi_{+}^{A}$, & $\psi_{-}^{\dot{A}}$, & \\
\hline
\end{tabular}

Table 1. Fields in the $2 \mathrm{D} \mathcal{N}=(4,4)$ theory.

hypermultiplet arises from D2-D6 strings. We reduce this theory to two dimensions, and denote the fields in the $2 \mathrm{D} \mathcal{N}=(4,4)$ theory as follows.

The $2 \mathrm{D}$ theory has $\mathrm{SU}(2)^{3}$ R-symmetry and an $\mathrm{SU}(2)$ flavor symmetry acting on the adjoint hypermultiplet. The indices $I, \dot{I}, A, \dot{A}$ are for the doublets under the symmetry $\mathrm{SU}(2)_{1}, \cdots, \mathrm{SU}(2)_{4}$, where $\mathrm{SU}(2)_{1}$ is the flavor symmetry and the other three are R-symmetries. The $\mathcal{N}=(4,4)$ supersymmetry is parametrized by the spinors $\xi_{+}^{\dot{I} \dot{A}}$ and $\xi_{-}^{\dot{I} A}$.

The NS5-branes provide the Dirichlet boundary condition on $Y^{A \dot{A}}, \lambda_{-}^{\dot{I} A}$ and $\Psi_{+}^{I A}$, but other fields obey Neumann boundary condition. They also break the supersymmetry parametrized by $\xi_{-}^{\dot{I} A}$.

In this construction, one can choose freely where to put the M2-M5 system in the transverse Taub-NUT geometry. This corresponds to the choice of boundary conditions $Y^{A \dot{A}}=$ $u^{A \dot{A}} \cdot \mathbf{1}_{(N \times N)}$. For generic nonzero $u^{A \dot{A}}$ the fundamental hypermultiplet fields become all massive. Also, the $\mathrm{SU}(2)_{3} \times \mathrm{SU}(2)_{4}$ symmetry is broken to a diagonal subgroup. In the limit of large $u^{A \dot{A}}$ the fundamental hypermultiplet gets frozen, and the resulting system of adjoint fields is actually the $\mathcal{N}=(4,4)$ super Yang-Mills theory with R-symmetry $\mathrm{SU}(2)_{1} \times$ $\mathrm{SU}(2)_{2} \times \mathrm{SU}(2)_{\text {diag }}$, with the supersymmetry parametrized by $\xi_{+}^{\dot{I} \dot{A}}$ and $\xi_{-}^{I \dot{A}}$. This theory is in agreement with the ABJM slab when the M2-M5 system is away from the origin of $\mathbb{R}_{(789 \text { Ł) }}^{4}$.

On the other hand, for $u^{A \dot{A}}=0$ the theory has only $\mathcal{N}=(0,4)$ supersymmetry but an enhanced global symmetry $\mathrm{SU}(2)^{4}$, since $\mathrm{SU}(2)_{3}$ and $\mathrm{SU}(2)_{4}$ become independent. As compared to the ABJM slab at $u_{A}=0$, the global symmetry matches but the supersymmetry does not agree.

Thus the two derivations of the worldsheet theory of self-dual strings, using ABJM and IIA brane models, led to considerably different results. Part of the difference, for example the mismatch of symmetry and SUSY, is because we broke the symmetry explicitly on the IIA side by replacing the background $\mathbb{R}^{4}$ by a Taub-NUT. If the two derivations are both valid, then there should be a smooth interpolation of the two models corresponding to changing the asymptotic radius $R$ of the circle fiber of the Taub-NUT. One can also check the validity by evaluating $R$-independent physical observables in the two descriptions and making comparison.

\subsection{Quiver models}

Let us briefly comment on the case with more than two M5-branes. Suppose there are $K$ M5-branes at the origin of $\mathbb{R}_{(789 \text { দ })}^{4}$ mutually separated in the $y$-direction. The $i$-th M5-brane is at $y=y_{i}$, where $y_{1}<y_{2}<\cdots<y_{K}$. In addition, we have $N_{i}$ M2-branes suspended in the $i$-th interval $\left[y_{i}, y_{i+1}\right]$. The corresponding IIA brane system is described in figure 1 . 


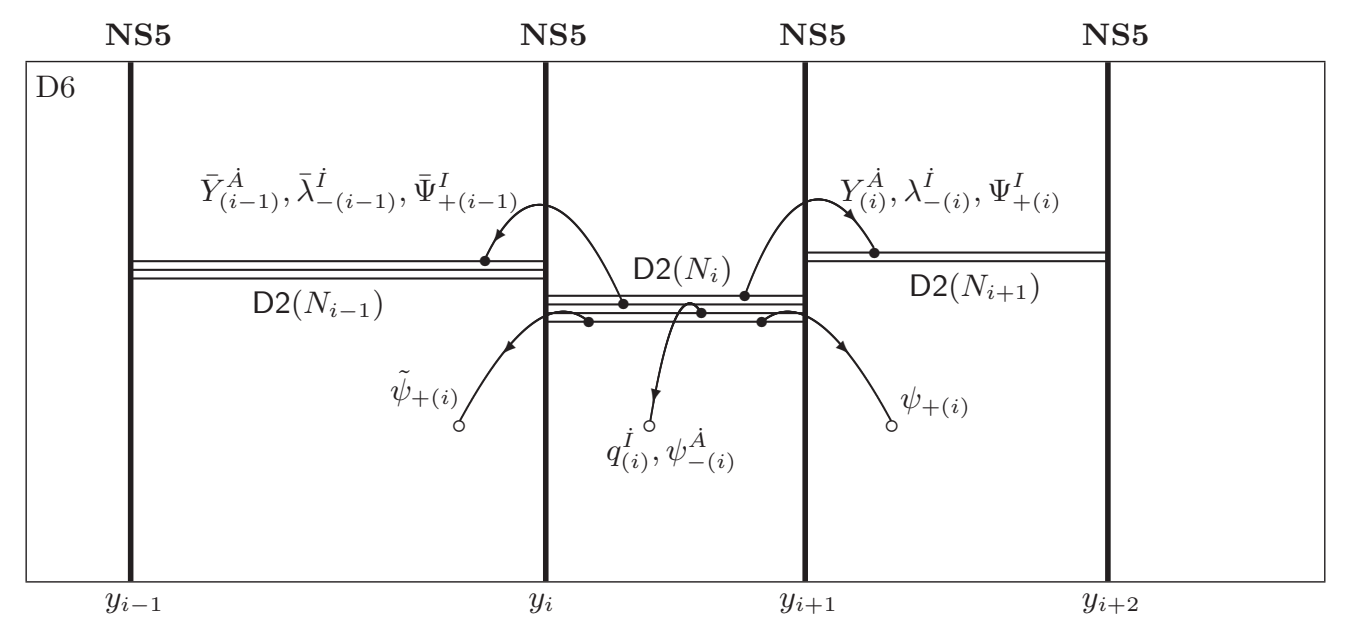

Figure 1. Type IIA brane system which gives rise to a quiver gauge theory. The D2-D2 strings and D2-D6 strings in this figure correspond to the bi-fundamental and fundamental matter fields, respectively.

From the type IIA brane picture, we obtain the following $2 \mathrm{D} \mathcal{N}=(0,4)$ SUSY gauge theory [2]. The gauge group is $\otimes_{i=1}^{K-1} \mathrm{U}\left(N_{i}\right)$, where the $\mathrm{U}\left(N_{i}\right)$ arises from the D2-branes in the $i$-th interval. Similarly to the model discussed in section 4.3, one has the adjoint and fundamental fields for each $\mathrm{U}\left(N_{i}\right)$,

$$
\begin{aligned}
& A_{\mu(i)}, Z_{(i)}^{I \dot{I}}, \Psi_{-(i)}^{I \dot{A}}, \lambda_{+(i)}^{\dot{I} \dot{A}}: \text { adjoint of } \mathrm{U}\left(N_{i}\right) \\
& q_{(i)}^{\dot{I}}, \psi_{-(i)}^{\dot{A}}, \psi_{+(i)}, \tilde{\psi}_{+(i)}: \quad \mathbf{N}_{i} \text { of } \mathrm{U}\left(N_{i}\right) .
\end{aligned}
$$

Here we denoted by $\psi_{+(i)}, \tilde{\psi}_{+(i)}$ the two components of the fundamental chiral spinor $\psi_{+}^{A}$ in the table 1 . In addition, there are bi-fundamental matter fields connecting the neighboring gauge groups,

$$
Y_{(i)}^{\dot{A}}, \lambda_{-(i)}^{\dot{I}}, \Psi_{+(i)}^{I}: \quad\left(\mathbf{N}_{i}, \overline{\mathbf{N}}_{i+1}\right) \text { of } \mathrm{U}\left(N_{i}\right) \times \mathrm{U}\left(N_{i+1}\right) .
$$

In this quiver theory the $\mathrm{SU}(2)_{3}$ is broken to $\mathrm{U}(1)$. Under this $\mathrm{U}(1)$, the bi-fundamental fields $Y_{(i)}^{\dot{A}}, \lambda_{-(i)}^{\dot{I}}, \Psi_{+(i)}^{I}$ carry the charge $1 / 2$, the fundamental fields $\left(\psi_{+(i)}, \tilde{\psi}_{+(i)}\right)$ carry $(+1 / 2,-1 / 2)$, and all other fields are neutral. These fields carrying nonzero U(1) charge all correspond to open strings connecting D-branes in the neighboring intervals, as shown in figure 1. The charge assignments to the fields and the symmetry breaking can be most easily understood by considering a similar system of branes with periodic identification of $x_{2}$ direction. After T-dualizing along $x_{2}$ we obtain the system of D1, D5-branes in a transverse $\mathbb{Z}_{K}$ orbifold, for which the standard construction [24] allows one to identify the worldvolume field theory.

To study the same system using ABJM model, one needs not only the boundary condition but also the junction condition on fields for M2-branes intersecting with M5branes. With regard to this aspect, our current understanding of the ABJM model is rather limited. The similar system of D3-branes ending on or intersecting with D5-branes 
was studied systematically in [8] through the analysis of Nahm equation and the moduli space of its solutions. There it was shown that the physics at the D3-D5 intersection varies very much depending on the number of D3-branes ending on the two sides of a D5-brane. The system of M2 and M5-branes needs to be studied in a similar manner.

\section{$5 \quad$ Elliptic genus}

In this section we study the elliptic genus for the two 2D gauge theories for self-dual strings. If the two theories are dual or connected by some continuous deformation, their elliptic genera should agree. Elliptic genus for general $2 \mathrm{D} \mathcal{N}=(2,2)$ and $\mathcal{N}=(0,2)$ supersymmetric gauge theories has been studied recently in $[6,7,25]$. On the other hand, the elliptic genus of multiple self-dual strings has been derived in [1] using topological vertex formalism.

Elliptic genus can be formulated for $2 \mathrm{D}$ theories with at least $\mathcal{N}=(0,2)$ supersymmetry as a partition function on a two-torus with SUSY preserving boundary condition on fields, and is a holomorphic function of the modulus $\tau$. If the theory has global symmetry that commutes with $\mathcal{N}=(0,2)$ supersymmetry, one can gauge it by an external flat gauge field $A_{\mu}$. Then the elliptic genus also depends holomorphically on the fugacity parameter $w \equiv \operatorname{Im} \tau \cdot\left(A_{1}+i A_{2}\right) / 2 \pi i$.

Constructions of 2D SUSY theories. We begin by summarizing how $2 \mathrm{D}$ field theories with various supersymmetry can be constructed from $\mathcal{N}=(0,2)$ supermultiplets. Gauge theories with $\mathcal{N}=(0,2)$ supersymmetry generally consist of three kinds of multiplets, namely vector multiplet $\left(A_{\mu}, \lambda_{+}, \bar{\lambda}_{+}, D\right)$,

$$
\begin{array}{rlrl}
\delta A_{1} & =-i \delta A_{2}=\xi_{+} \bar{\lambda}_{+}+\bar{\xi}_{+} \lambda_{+}, & & \delta \lambda_{+}=\xi_{+}\left(i F_{12}+D\right), \\
\delta D & =\left(D_{1}+i D_{2}\right)\left(\xi_{+} \bar{\lambda}_{+}-\bar{\xi}_{+} \lambda_{+}\right), & \delta \bar{\lambda}_{+}=\bar{\xi}_{+}\left(i F_{12}-D\right),
\end{array}
$$

chiral multiplet $\left(q, \psi_{-}\right)$with its conjugate anti-chiral multiplet $\left(\bar{q}, \bar{\psi}_{-}\right)$,

$$
\begin{array}{ll}
\delta q=2 \xi_{+} \psi_{-}, & \delta \psi_{-}=-\bar{\xi}_{+}\left(D_{1}+i D_{2}\right) q, \\
\delta q=2 \bar{\xi}_{+} \bar{\psi}_{-}, & \delta \bar{\psi}_{-}=-\xi_{+}\left(D_{1}+i D_{2}\right) \bar{q},
\end{array}
$$

and Fermi multiplet $\left(\psi_{+}, F ; \Phi\right)$ with its conjugate $\left(\bar{\psi}_{+}, \bar{F} ; \bar{\Phi}\right)$,

$$
\begin{array}{ll}
\delta \psi_{+}=\xi_{+} F+\bar{\xi}_{+} \Phi, & \delta F=-2 \bar{\xi}_{+}\left(D_{1}+i D_{2}\right) \psi_{+}-2 \bar{\xi}_{+} \Psi_{-}, \\
\delta \bar{\psi}_{+}=-\bar{\xi}_{+} \bar{F}+\xi_{+} \bar{\Phi}, & \delta \bar{F}=2 \xi_{+}\left(D_{1}+i D_{2}\right) \bar{\psi}_{+}+2 \xi_{+} \bar{\Psi}_{-} .
\end{array}
$$

Here $\left(\Phi, \Psi_{-}\right)$is a chiral multiplet made of fields sitting in other multiplets. The kinetic terms for these multiplets are

$$
\begin{aligned}
& \mathcal{L}_{\text {vec }}=\operatorname{Tr}\left(F_{12}^{2}+D^{2}-2 \bar{\lambda}_{+}\left(D_{1}+i D_{2}\right) \lambda_{+}\right), \\
& \mathcal{L}_{\text {chi }}=D_{\mu} \bar{q} D_{\mu} q+2 \bar{\psi}_{-}\left(D_{1}-i D_{2}\right) \psi_{-}+i \bar{q} D q-2 i \bar{\psi}_{-} \bar{\lambda}_{+} q-2 i \bar{q} \lambda_{+} \psi_{-}, \\
& \mathcal{L}_{\text {fer }}=\bar{\Phi} \Phi+\bar{F} F-2 \bar{\psi}_{+}\left(D_{1}+i D_{2}\right) \psi_{+}+2 \bar{\Psi}_{-} \psi_{+}-2 \bar{\psi}_{+} \Psi_{-} .
\end{aligned}
$$

All these are SUSY exact. As usual, vector multiplet fields are regarded as matrices and chiral (anti-chiral) fields are regarded as column (row) vectors. Interactions can be 
introduced as an $F$-component of a gauge invariant Fermi multiplet with vanishing $\Phi$ component. For example, consider some chiral multiplets $\left(J^{(i)}, \Xi^{(i)}\right)$ and some Fermi multiplets $\left(\psi_{+}^{(i)}, F^{(i)} ; \Phi^{(i)}\right)$. Then $\sum_{i} \psi_{+}^{(i)} J^{(i)}$ is the lowest component of a Fermi multiplet. From its $F$-component one obtains

$$
\mathcal{L}_{\text {int }}=i \sum_{i}\left(F^{(i)} J^{(i)}+\bar{J}^{(i)} \bar{F}^{(i)}-2 \psi_{+}^{(i)} \Xi_{-}^{(i)}-2 \bar{\Xi}_{-}^{(i)} \bar{\psi}_{+}^{(i)}\right),
$$

which is supersymmetric if $\sum_{i} \Phi^{(i)} J^{(i)}=0$. Another example is the FI-theta term for abelian vector multiplets, which is indeed the $F$-component of a Fermi multiplet starting from $\lambda_{+}$.

$\mathcal{N}=(2,2)$ vector multiplet is obtained by combining an $\mathcal{N}=(0,2)$ vector multiplet $\left(A_{\mu}, \lambda_{+}, \bar{\lambda}_{+}, D\right)$ and an adjoint chiral multiplet $\left(Y, \lambda_{-}\right)$. Likewise, an $\mathcal{N}=(2,2)$ chiral multiplet is obtained by combining a chiral multiplet $\left(q, \psi_{-}\right)$and a Fermi multiplet $\left(\psi_{+}, F ; \Phi=i Y q\right)$ in the same representation of the gauge group. The kinetic Lagrangian is given by a sum of those in (5.4). To construct $\mathcal{L}_{\text {int }}$, one chooses a gauge invariant function $W\left(q^{(i)}\right)$ of chiral fields as superpotential and set $J^{(i)}=\partial W / \partial q^{(i)}$ in (5.5).

$\mathcal{N}=(4,4)$ vector multiplet consists of an $\mathcal{N}=(2,2)$ vector multiplet $\left(A_{\mu}, Y, \lambda, \bar{\lambda}, D\right)$ and an adjoint chiral multiplet $(\tilde{Y}, \tilde{\lambda}, F)$. A hypermultiplet is made of a pair of $\mathcal{N}=$ $(2,2)$ chiral multiplets, with lowest components $q, \tilde{q}$, sitting in conjugate representations of gauge group. The Lagrangian is uniquely determined from the gauge symmetry and its representation. In particular we need to introduce a specific superpotential $W=\tilde{q} \tilde{Y} q$ to have $\mathcal{N}=(4,4)$ supersymmetry.

Elliptic genus. Let us next introduce a powerful formula for elliptic genus of $\mathcal{N}=(0,2)$ supersymmetric gauge theories obtained in $[6,7]$. Their derivation was based on localization of path integral, and the final formula is expressed in terms of the so-called Jeffrey-Kirwan residue.

For $\mathcal{N}=(0,2)$ theories on torus, all the SUSY invariants one can use for Lagrangian are actually SUSY exact. For theories with standard kinetic Lagrangians (5.4), the elliptic genus can be computed using SUSY localization $[6,7]$ and the result basically depends only on the field content and symmetry.

Due to supersymmetry, the path integral localizes onto the moduli space of BPS configurations, which in this case is the moduli space of flat gauge fields. For rank- $r$ gauge group, the moduli space is real $2 r$-dimensional and is parametrized by $r$ complex coordinates $w_{1}, \cdots, w_{r}$ with periodicity $w_{i} \sim w_{i}+1 \sim w_{r}+\tau$. At first glance, we seem to obtain an integral $\mathrm{d}^{r} w \mathrm{~d}^{r} \bar{w}$ of some one-loop determinant which is meromorphic in $w_{i}$. However, a proper treatment of the zeromodes of the gaugino $\lambda_{+}, \bar{\lambda}_{+}$brings in a $\bar{w}_{i}$-dependence. It was shown in $[6,7]$ that, when this effect is combined with a nice regularization of the divergence of the determinant, the $2 r$-dimensional integral can be transformed into an integral of a certain $(r, 0)$-form $Z_{1 \text {-loop }}$ by a repeated use of Stokes theorem. The elliptic genus thus becomes the sum of the so-called Jeffrey-Kirwan residues of $Z_{1 \text {-loop }}$ at all the "poles" $w_{*}$ in the moduli space where $r$ or more singular hypersurfaces intersect.

$$
Z_{T^{2}}(\tau)=\frac{1}{|W|} \sum_{w_{*}} \underset{\substack{\mathrm{JK}-\operatorname{Res}_{w} \\ w=w_{*}}}{\operatorname{Rol})} Z_{1 \text {-loop }}(\tau, w) .
$$


Here $|W|$ is the order of the Weyl group, and $\eta$ is a real $r$-component vector we need to choose to define the residue operation. The final result for $Z_{T^{2}}$ is independent of the choice of $\eta$. The $(r, 0)$-form $Z_{1 \text {-loop }}$ is given by the product of contributions from the vector, chiral and Fermi multiplets,

$$
\begin{aligned}
\Delta_{\text {vec }} & =\left(\frac{2 \pi \eta(\tau)^{2}}{i}\right)^{r} \prod_{\alpha: \text { roots }} \frac{i \theta_{1}(\tau \mid \alpha \cdot w)}{\eta(\tau)} \mathrm{d}^{r} w, \\
\Delta_{\text {chi }} & =\prod_{\lambda: \text { weights }} \frac{i \eta(\tau)}{\theta_{1}(\tau \mid \lambda \cdot w)}, \\
\Delta_{\text {fer }} & =\prod_{\lambda: \text { weights }} \frac{i \theta_{1}(\tau \mid \lambda \cdot w)}{\eta(\tau)},
\end{aligned}
$$

where $\theta_{1}$ is Jacobi theta function. It should be obvious how the $Z_{T^{2}}$ will depend on additional parameters corresponding to external flat gauge fields coupled to global symmetries.

Jeffrey-Kirwan residue formula. We next present some defining properties of the Jeffrey-Kirwan residue operation which will be used in section 5.2. A more detailed definition is given in [7], see also [26, 27].

Consider $n(\geq r)$ singular hyperplanes in $\mathbb{R}^{r}$ meeting at the origin, defined by

$$
Q_{i} \cdot w=0 . \quad(i=1, \cdots, n)
$$

We denote the ordered set of charges by $\Delta=\left\{Q_{1}, \cdots, Q_{n}\right\}$. In the computation of elliptic genus, $Q_{i}$ are the weight vectors of the $n$ matter chiral fields which acquire zeromodes at $w=0$. In the case $n=r$, the Jeffrey-Kirwan residue is defined by the property

$$
\underset{w=0}{\operatorname{JK}-\operatorname{Res}}(\eta) \frac{\mathrm{d}^{r} w}{\left(Q_{1} \cdot w\right) \cdots\left(Q_{r} \cdot w\right)}= \begin{cases}\frac{1}{\left|\operatorname{det}\left(Q_{1} \cdots Q_{r}\right)\right|} & \text { if } \eta \in \operatorname{Cone}\left(Q_{1}, \cdots, Q_{r}\right) \\ 0 & \text { otherwise. }\end{cases}
$$

Namely, it vanishes unless $\eta$ is expressed as a linear combination of $\left\{Q_{1}, \cdots, Q_{r}\right\}$ with positive coefficients. Note that one should keep track of the charge vectors $\left\{Q_{i}\right\}$ including their sign in the residue computation.

When $n>r$, there is a number of ways to choose from $\Delta$ an ordered set of $r$ linearly independent charge vectors $b=\left\{Q_{i_{1}}, \cdots, Q_{i_{r}}\right\}_{\left(i_{1}<\cdots<i_{r}\right)}$, which we call a basis of $\Delta$. To each basis $b$ of $\Delta$ one can associate a basic fraction

$$
\phi_{b} \equiv \frac{1}{\prod_{Q_{i} \in b} Q_{i} \cdot w}=\frac{1}{\left(Q_{i_{1}} \cdot w\right) \cdots\left(Q_{i_{r}} \cdot w\right)} .
$$

The basic fractions thus obtained may obey some linear relations. For example, from the set of three charge vectors $\Delta=\{(1,0),(0,1),(1,1)\}$ one gets three basic fractions

$$
\phi_{b_{1}}=\frac{1}{w_{1} w_{2}}, \quad \phi_{b_{2}}=\frac{1}{w_{1}\left(w_{1}+w_{2}\right)}, \quad \phi_{b_{3}}=\frac{1}{w_{2}\left(w_{1}+w_{2}\right)}
$$

obeying one relation $\phi_{b_{1}}=\phi_{b_{2}}+\phi_{b_{3}}$. In this example the basic fractions form a 2dimensional vector space, and any pair of basic fractions can be used as a basis. We 
denote by $B$ a set of bases of $\Delta$ such that $\left\{\phi_{b}\right\}_{b \in B}$ form a basis of basic fractions. The Jeffrey-Kirwan residue of basic fractions is given by the formula (5.9), whereas the fractions whose denominators do not contain $r$ linearly independent factors, for example

$$
\frac{1}{w_{1}}, \frac{1}{\left(w_{1}+w_{2}\right)^{2}}, \cdots
$$

have trivial residues. More general meromorphic functions can all be decomposed into derivatives of basis basic fractions and fractions with trivial residue. For example,

$$
\frac{1}{w_{1} w_{2}\left(w_{1}+w_{2}\right)}=-\frac{\partial}{\partial w_{1}} \frac{1}{w_{1} w_{2}}+\left(\frac{\partial}{\partial w_{1}}-\frac{\partial}{\partial w_{2}}\right) \frac{1}{w_{1}\left(w_{2}+w_{2}\right)} .
$$

Therefore, the Jeffrey-Kirwan residue is uniquely determined by its value on a basis of basic fractions.

Residue integrals can be regarded as linear functions on the space of basic fractions. In the general case $n>r$ there are a number of $r$-cycles to define residue integrals, and they are subject to some linear relations. Here we quote from [27] a useful proposition. For any $\Delta$, there is a choice of $B$ such that the set of iterated residues $\operatorname{Res}_{b}(b \in B)$

$$
\operatorname{Res}_{b} \equiv \underset{Q_{i_{r}} \cdot w=0}{\operatorname{Res}} \cdots \operatorname{Res}_{Q_{i_{1}} \cdot w=0}^{\operatorname{Res}} \quad\left(b=\left\{Q_{i_{1}}, \cdots, Q_{i_{r}}\right\}_{\left(i_{1}<\cdots<i_{r}\right)} \in B\right)
$$

form a dual basis to the basis of basic fractions $\left\{\phi_{b}\right\}_{b \in B}$, namely $\operatorname{Res}_{b} \phi_{b^{\prime}}=\delta_{b b^{\prime}}$. The iterated residue above means one first takes the residue along the first hyperplane $Q_{i_{1}} \cdot w=0$ keeping other $r-1$ variables fixed and generic, then takes the residue along the second hyperplane $Q_{i_{2}} \cdot w=0$, and goes on. Note that the order of the iterated residue is determined according to the order of the charges in $\Delta$ we have chosen (arbitrarily) at the beginning. With respect to this choice of $B$, the Jeffrey-Kirwan residue is simply

$$
\operatorname{Res}(\eta)=\sum_{b \in B ; \eta \in \operatorname{Cone}(b)} \nu(b) \cdot \operatorname{Res}_{b},
$$

where $\nu(b)= \pm 1$ is the orientation of the basis $b$.

\subsection{IIA brane model}

We first study the elliptic genus for the IIA brane model. As was explained in section 4.3, the theory can be obtained from a $2 \mathrm{D} \mathcal{N}=(4,4)$ supersymmetric gauge theory by freezing some of the fields.

The $\mathcal{N}=(4,4)$ system is a $\mathrm{U}(N)$ gauge theory with one adjoint and one fundamental hypermultiplets. The fields and their $J^{3}$ charges under the global symmetry $\mathrm{SU}(2)^{4}$ are listed in table 2. According to the charge assignments given there, the parameter of $\mathcal{N}=$ $(0,2)$ supersymmetry $\xi_{+}$carries the charges $\left(J_{(1)}^{3}, J_{(2)}^{3}, J_{(3)}^{3}, J_{(4)}^{3}\right)=\left(0, \frac{1}{2}, 0,-\frac{1}{2}\right)$. The $\mathcal{N}=$ $(0,4)$ system of our interest is obtained by freezing the multiplets including $Y, \tilde{Y}, \Psi_{+}$and $\tilde{\Psi}_{+}$. If we turn on a nonzero classical value $\langle Y\rangle=u \cdot \mathbf{1}_{(N \times N)}$ in this process, the symmetry $\mathrm{SU}(2)_{3} \times \mathrm{SU}(2)_{4}$ is broken to a diagonal $\mathrm{SU}(2)$ which contains $J_{(3)}^{3}+J_{(4)}^{3}$.

In the standard approach reviewed above, the elliptic genus is given by an integral with respect to the moduli of flat $\mathrm{U}(N)$ gauge fields on torus which we denote by $\left(w_{1}, \cdots, w_{N}\right)$. 


\begin{tabular}{|c|c|c|c|c|c|c|}
\hline \multirow[b]{2}{*}{$\mathcal{N}=(4,4)$} & \multirow[b]{2}{*}{$\mathcal{N}=(2,2)$} & \multirow[b]{2}{*}{ rep } & \multicolumn{2}{|c|}{$\mathcal{N}=(0,2)$ chiral } & \multicolumn{2}{|c|}{$\mathcal{N}=(0,2)$ Fermi } \\
\hline & & & (scalar) & (spinor) & (spinor) & (vector) \\
\hline \multirow{4}{*}{ vector } & \multirow{2}{*}{ vector } & \multirow{2}{*}{ adj } & $Y(\cdot \cdot+-)$ & $\bar{\lambda}_{-}(\cdot-+\cdot)$ & $\lambda_{+}(\cdot+\cdot-)$ & $A_{\mu}$ \\
\hline & & & $\bar{Y}(\cdot-+)$ & $\lambda_{-}(\cdot+-\cdot)$ & $\bar{\lambda}_{+}(\cdot-\cdot+)$ & \\
\hline & \multirow{2}{*}{ chiral } & \multirow{2}{*}{ adj } & $\tilde{Y}(\cdot \cdot--)$ & $\tilde{\lambda}_{-}(\cdot--\cdot)$ & $\tilde{\lambda}_{+}(\cdot-\cdot-)$ & \\
\hline & & & $\overline{\tilde{Y}}(\cdots++)$ & $\overline{\tilde{\lambda}}_{-}(\cdot++\cdot)$ & $\overline{\tilde{\lambda}}_{+}(\cdot+\cdot+)$ & \\
\hline \multirow{4}{*}{ hyper } & \multirow{2}{*}{ chiral } & adj & $Z(++\cdot \cdot)$ & $\Psi_{-}(+\cdot \cdot+)$ & $\Psi_{+}(+\cdot+\cdot)$ & \\
\hline & & adj & $\bar{Z}(--\cdot \cdot)$ & $\bar{\Psi}_{-}(-\cdot-)$ & $\bar{\Psi}_{+}(-\cdot-\cdot)$ & \\
\hline & \multirow{2}{*}{ chiral } & $\operatorname{adj}$ & $\tilde{Z}(-+\cdot \cdot)$ & $\tilde{\Psi}_{-}(-\cdot++)$ & $\tilde{\Psi}_{+}(-\cdot+\cdot)$ & \\
\hline & & adj & $\tilde{\tilde{Z}}(+-\cdot \cdot)$ & $\tilde{\Psi}_{-}(+\cdot \cdot-)$ & $\tilde{\Psi}_{+}(+\cdot-\cdot)$ & \\
\hline \multirow{4}{*}{ hyper } & \multirow{2}{*}{ chiral } & $\square$ & $q(\cdot+\cdot \cdot)$ & $\psi_{-}(\cdot \cdot+)$ & $\psi_{+}(\cdot+\cdot)$ & \\
\hline & & $\bar{\square}$ & $\bar{q}(\cdot-\cdot \cdot)$ & $\bar{\psi}_{-}(\cdot \cdot-)$ & $\bar{\psi}_{+}(\cdot-\cdot)$ & \\
\hline & \multirow{2}{*}{ chiral } & $\square$ & $\tilde{q}(\cdot+\cdot \cdot)$ & $\tilde{\psi}_{-}(\cdots+)$ & $\tilde{\psi}_{+}(\cdot+\cdot)$ & \\
\hline & & $\square$ & $\overline{\tilde{q}}(\cdot-\cdot \cdot)$ & $\tilde{\psi}_{-}(\cdot \cdot-)$ & $\tilde{\bar{\psi}}_{+}(\cdot \cdot-\cdot)$ & \\
\hline
\end{tabular}

Table 2. The fields of an $\mathcal{N}=(4,4)$ SUSY theory with the $J^{3}$ eigenvalues of the four SU(2) global symmetries. The symbol $(\cdot++)$ means $\left(J_{(1)}^{3}, J_{(2)}^{3}, J_{(3)}^{3}, J_{(4)}^{3}\right)=\left(0,0, \frac{1}{2},-\frac{1}{2}\right)$. In the $\mathcal{N}=(0,4)$ supersymmetric system of our interest, the fields in the multiplets including $Y, \tilde{Y}, \Psi_{+}, \tilde{\Psi}_{+}$are frozen.

In addition, we gauge the $\mathrm{U}(1)^{4}$ symmetry generated by $J_{(1)}^{3}, \cdots, J_{(4)}^{3}$ by external flat gauge fields. The corresponding fugacity parameters are denoted as follows,

$$
w_{\mathrm{bg}(1)}=\epsilon_{1}-\epsilon_{2}, \quad w_{\mathrm{bg}(2)}=\epsilon_{1}+\epsilon_{2}, \quad w_{\mathrm{bg}(3)}=2 m, \quad w_{\mathrm{bg}(4)}=\epsilon_{1}+\epsilon_{2} .
$$

Note that we need $w_{\mathrm{bg}(2)}=w_{\mathrm{bg}(4)}$ in order to preserve the $\mathcal{N}=(0,2)$ supersymmetry. Using (5.7) one finds that the meromorphic form $Z_{1 \text {-loop }}$ is given by

$$
\begin{aligned}
Z_{1-\text { loop }}= & \mathrm{d}^{N} w \times \theta_{1}^{\prime N} \prod_{i \neq j} \theta_{1}\left(w_{i}-w_{j}\right) \prod_{i, j} \frac{\theta_{1}\left(w_{i}-w_{j}+\epsilon_{1}+\epsilon_{2}\right)}{\theta_{1}\left(w_{i}-w_{j}+\epsilon_{1}\right) \theta_{1}\left(w_{i}-w_{j}+\epsilon_{2}\right)} \\
& \times \prod_{i} \frac{\theta_{1}\left(w_{i}-m\right) \theta_{1}\left(-w_{i}-m\right)}{\theta_{1}\left(w_{i}-\frac{1}{2}\left(\epsilon_{1}+\epsilon_{2}\right)\right) \theta_{1}\left(-w_{i}-\frac{1}{2}\left(\epsilon_{1}+\epsilon_{2}\right)\right)} .
\end{aligned}
$$

Here and in the following, the first argument of $\theta_{1}$ function is omitted since it is always $\tau$, and $\theta_{1}^{\prime}=2 \pi \eta(\tau)^{3}$ is the $w$-derivative of $\theta_{1}(\tau \mid w)$ at $w=0$. If one wishes to turn on a classical value of the adjoint scalar $Y$, an additional condition $w_{\mathrm{bg}(3)}=w_{\mathrm{bg}(4)}$ or $2 m=\epsilon_{1}+\epsilon_{2}$ is needed due to symmetry breaking.

The evaluation of the Jeffrey-Kirwan residue seems quite intricate, so we propose an alternative way to compute the elliptic genus based on the idea of Higgs branch localization. This was proposed in the study of $S^{2}$ partition function of 2D SUSY gauge theories in [28, 29], and generalized to problems in higher dimensions in [30-34]. In our problem, this basically requires the BPS configurations to satisfy also the F-term and D-term constraints 
arising from the equation of motion of the auxiliary fields in $\mathcal{N}=(0,4)$ vector multiplet. For the theory of our interest, the constraints are given by

$$
\begin{aligned}
q \bar{q}-\overline{\tilde{q}} \tilde{q}+[Z, \bar{Z}]+[\tilde{Z}, \overline{\tilde{Z}}]+\zeta \cdot \mathbf{1}_{(N \times N)} & =0 \\
q \tilde{q}+[Z, \tilde{Z}] & =0
\end{aligned}
$$

where we have turned on a FI deformation $\zeta$. In fact, these are nothing but the ADHM equation for the moduli space of $N \mathrm{U}(1)$-instantons. This is as expected since the D2-branes within a D6-brane are known to behave like $\mathrm{U}(1)$ instantons.

The constraints (5.17) and the $\mathrm{U}(N)$ gauge equivalence determine the moduli space of vacua of the theory. For nonzero $\zeta$, some scalar fields must condense and break the gauge symmetry completely. In the IR the theory flows to a non-linear sigma model on the moduli space of $N \mathrm{U}(1)$-instantons, and what we are after is the elliptic genus for that sigma model. In this sigma model the fugacity parameters $\epsilon_{1}, \epsilon_{2}, m$ enter through the gauging of certain isometry of the target space. In particular, there are a finite number of points on the target space fixed under the isometry corresponding to $\epsilon_{1}, \epsilon_{2}$. At each fixed point the sigma model is well approximated by a free theory of chiral and Fermi multiplets coupled to some external flat gauge fields. The elliptic genus for the sigma model is obtained by summing over those fixed point contributions.

This consideration leads us to propose the following formula for the elliptic genus

$$
\begin{aligned}
Z_{\mathrm{IIA}}=\sum_{\left\{w_{i}\right\}} \prod_{i, j} \frac{\theta_{1}\left(w_{i}-w_{j}\right) \theta_{1}\left(w_{i}-w_{j}+\epsilon_{1}+\epsilon_{2}\right)}{\theta_{1}\left(w_{i}-w_{j}+\epsilon_{1}\right) \theta_{1}\left(w_{i}-w_{j}+\epsilon_{2}\right)} \\
\cdot \prod_{i} \frac{\theta_{1}\left(w_{i}-m\right) \theta_{1}\left(-w_{i}-m\right)}{\theta_{1}\left(w_{i}-\frac{1}{2}\left(\epsilon_{1}+\epsilon_{2}\right)\right) \theta_{1}\left(-w_{i}-\frac{1}{2}\left(\epsilon_{1}+\epsilon_{2}\right)\right)} .
\end{aligned}
$$

Here the sum is over all the fixed points in Higgs branch, which are all characterized by the value of flat $\mathrm{U}(N)$ gauge field $\left\{w_{i}\right\}$. At each of the fixed points, $\left\{w_{i}\right\}$ must be chosen appropriately so that some scalars have zeromodes there and condense. The summand in the above formula is the one-loop determinant without excluding the gaugino zeromodes. It has therefore $N$ obvious zeroes $\theta_{1}\left(w_{i}-w_{i}\right)$ in the enumerator, but at each fixed point we anticipate $N$ zeroes appear in the denominator to cancel them. The formula (5.18) makes sense and gives a finite value once this cancellation is understood.

To determine the value of $\left\{w_{i}\right\}$ we notice that, in terms of the UV gauge theory variables, the fixed points are described by the solutions of (5.17) and the BPS condition

$$
\begin{aligned}
& \mathbf{w} Z-Z \mathbf{w}+\epsilon_{1} Z=0, \quad \mathbf{w} q+\frac{1}{2}\left(\epsilon_{1}+\epsilon_{2}\right) q=0, \\
& \mathbf{w} \tilde{Z}-\tilde{Z} \mathbf{w}+\epsilon_{2} \tilde{Z}=0, \quad-\tilde{q} \mathbf{w}+\frac{1}{2}\left(\epsilon_{1}+\epsilon_{2}\right) \tilde{q}=0,
\end{aligned}
$$

where $\mathbf{w} \equiv \operatorname{diag}\left(w_{1}, \cdots, w_{N}\right)$. It is an elementary math problem to solve the combined system of equations (5.17) and (5.19). See [35] for a detailed explanation. For example, for $\zeta>0$ one can first show that $\tilde{q}$ must be nonzero while $q=[Z, \tilde{Z}]=0$. Then one finds there are $N$ linearly independent row vectors of the form $\tilde{q} Z^{m} \tilde{Z}^{n}$ with the eigenvalue $\mathbf{w}=\left(m+\frac{1}{2}\right) \epsilon_{1}+\left(n+\frac{1}{2}\right) \epsilon_{2}$. The eigenvalue spectrum is thus described by a Young 
diagram, so the fixed points are labeled by a Young diagram of $N$ boxes. The precise value of the scalar fields at the fixed points is not important. But a careful look at the solution shows that, among $2 N^{2}+2 N$ components of scalar fields $(Z, \tilde{Z}, q, \tilde{q})$, there are precisely $N$ components acquiring nonzero values at every fixed point.

To find out the free theory that approximates the sigma model at each fixed point and compute its elliptic genus, we simply substitute the eigenvalues of $\mathbf{w}$ into the summand of (5.18). Since $\left\{w_{i}\right\}$ have been chosen so that $N$ scalars can condense, the denominator of the summand in (5.18) gives rise to $N$ zeroes that precisely cancel the $N$ zeroes in the enumerator, as expected. This is only a part of the manifestation of super Higgs mechanism; in fact, many similar cancellations occur for theta functions with nonzero arguments as well. The contribution from each fixed point can be evaluated using the formula [36]

$$
\begin{aligned}
\sum_{(k, l) \in \mathbf{Y}} t_{1}^{k} t_{2}^{l} & +\sum_{\left(k^{\prime}, l^{\prime}\right) \in \mathbf{Y}^{\prime}} t_{1}^{1-k^{\prime}} t_{2}^{1-l^{\prime}}-\sum_{(k, l) \in \mathbf{Y},\left(k^{\prime}, l^{\prime}\right) \in \mathbf{Y}^{\prime}} t_{1}^{k-k^{\prime}} t_{2}^{l-l^{\prime}}\left(1-t_{1}\right)\left(1-t_{2}\right) \\
& =\sum_{(k, l) \in \mathbf{Y}} t_{1}^{k-\tilde{\lambda}_{l}\left(\mathbf{Y}^{\prime}\right)} t_{2}^{1+\lambda_{k}(\mathbf{Y})-l}+\sum_{\left(k^{\prime}, l^{\prime}\right) \in \mathbf{Y}^{\prime}} t_{1}^{1+\tilde{\lambda}_{l^{\prime}}(\mathbf{Y})-k^{\prime}} t_{2}^{l^{\prime}-\lambda_{k^{\prime}}\left(\mathbf{Y}^{\prime}\right)},
\end{aligned}
$$

with the sum of monomials translated into the product of theta functions. Here $(k, l) \in \mathbf{Y}$ is a pair of positive integers labeling a box in the diagram $\mathbf{Y}$, and $\lambda_{k}(\mathbf{Y}), \tilde{\lambda}_{l}(\mathbf{Y})$ are the lengths of its $k$-th column and $l$-th row. Thus the contribution to the elliptic genus from the fixed point labeled by a Young diagram $\mathbf{Y}$ is

$$
Z_{\mathbf{Y}}=\prod_{(i, j) \in \mathbf{Y}} \frac{\theta_{1}\left(-m+\left(i-\frac{1}{2}\right) \epsilon_{1}+\left(j-\frac{1}{2}\right) \epsilon_{2}\right) \cdot \theta_{1}\left(-m-\left(i-\frac{1}{2}\right) \epsilon_{1}-\left(j-\frac{1}{2}\right) \epsilon_{2}\right)}{\theta_{1}\left(\left(i-\tilde{\lambda}_{j}\right) \epsilon_{1}+\left(\lambda_{i}-j+1\right) \epsilon_{2}\right) \cdot \theta_{1}\left(\left(\tilde{\lambda}_{j}-i+1\right) \epsilon_{1}+\left(j-\lambda_{i}\right) \epsilon_{2}\right)} .
$$

This formula can be interpreted as the elliptic genus of a free theory of $2 N$ chiral multiplets and $2 N$ Fermi multiplets coupled to some background flat gauge field, and the number $2 N$ agrees with the complex dimension of the Higgs branch moduli space. The elliptic genus of the IIA brane model is finally given by

$$
Z_{\mathrm{IIA}}=\sum_{\mathbf{Y}} Z_{\mathbf{Y}}
$$

This agrees with the result obtained from topological vertex formalism [1].

So far we have been considering the case with $\langle Y\rangle=\langle\tilde{Y}\rangle=0$. If $2 m=\epsilon_{1}+\epsilon_{2}$, one can turn on $\langle Y\rangle$ and make the fundamental fields massive. The elliptic genus for such theory should be independent of the mass of fundamental matters. In the limit $\langle Y\rangle \rightarrow \infty$ the fundamental fields are frozen and we are left with $\mathcal{N}=(4,4) \mathrm{U}(N)$ super Yang-Mills theory. Note that, as explained in section 4.3 , the $\mathcal{N}=(4,4)$ enhanced SUSY here is different from the one used to classify the fields in table 2 , and that the fields $A_{\mu}, Z, \tilde{Z}$ form the $\mathcal{N}=(4,4)$ vector multiplet together with the fermions. Since all the remaining degrees of freedom are in the adjoint, the $\mathrm{U}(1)$ part of the vector multiplet becomes free, and in particular has fermion zeromodes. The contribution to the elliptic genus from the U(1) part is identified with $Z_{\mathrm{IIA}}$ for the case $N=1$. Before setting $2 m=\epsilon_{1}+\epsilon_{2}$ it is given by [1]

$$
Z_{\mathrm{IIA}(N=1)}=\frac{\theta_{1}\left(m+\frac{1}{2} \epsilon_{1}+\frac{1}{2} \epsilon_{2}\right) \theta_{1}\left(m-\frac{1}{2} \epsilon_{1}-\frac{1}{2} \epsilon_{2}\right)}{\theta_{1}\left(\epsilon_{1}\right) \theta_{1}\left(\epsilon_{2}\right)} .
$$




\begin{tabular}{|cc|cc|}
\hline \multicolumn{2}{|c|}{$\mathcal{N}=(0,2)$ chiral } & \multicolumn{2}{c|}{$\mathcal{N}=(0,2)$ Fermi } \\
(scalar) & (spinor) & (spinor) & (vector) \\
\hline$Y(\cdot \cdots \cdot)$ & $\bar{\lambda}_{-}(\cdot-\cdot+)$ & $\lambda_{+}(\cdot+\cdot-)$ & $A_{\mu}$ \\
$\bar{Y}(\cdot \cdots \cdot)$ & $\lambda_{-}(\cdot+\cdot-)$ & $\bar{\lambda}_{+}(\cdot-\cdot+)$ & \\
\hline$Z(++\cdot \cdot)$ & $\Psi_{-}(+\cdot \cdot+)$ & $\Psi_{+}(\cdot++\cdot)$ \\
$\bar{Z}(--\cdot \cdot)$ & $\bar{\Psi}_{-}(-\cdot \cdot-)$ & $\bar{\Psi}_{+}(\cdot--\cdot)$ \\
\hline$\tilde{Z}(-+\cdot \cdot)$ & $\tilde{\Psi}_{-}(-\cdot \cdot+)$ & $\tilde{\Psi}_{+}(\cdot-+\cdot)$ \\
$\bar{Z}(+-\cdot \cdot)$ & $\overline{\tilde{\Psi}}_{-}(+\cdot \cdot-)$ & $\overline{\tilde{\Psi}}_{+}(\cdot+-\cdot)$ \\
\hline
\end{tabular}

Table 3. The fields of the dimensionally reduced ABJM model at $u=0$ with the $J^{3}$ eigenvalues of the four $\mathrm{SU}(2)$ global symmetries. All fields are in the adjoint of the gauge group $\mathrm{U}(N)$.

Factoring this out from $Z_{\text {IIA }}$ and setting $m=\frac{1}{2}\left(\epsilon_{1}+\epsilon_{2}\right)$, we should obtain the elliptic genus for $\mathcal{N}=(4,4) \mathrm{SU}(N)$ super Yang-Mills theory.

$$
\left.\frac{Z_{\mathrm{IIA}}}{Z_{\mathrm{IIA}(N=1)}}\right|_{m=\frac{1}{2}\left(\epsilon_{1}+\epsilon_{2}\right)}=Z_{\mathrm{SU}(N)} \text {. }
$$

\subsection{ABJM slab}

Next we study the elliptic genus for the theory (4.12) obtained from the dimensional reduction of the ABJM model. Although the importance of KK modes was emphasized in section 4.2, those massive modes can be safely neglected for the computation of the elliptic genus. Also, we set here $k=1$ to avoid the complication with the $\mathbb{Z}_{k}$ orbifolding.

The matter fields, namely $Z$ 's and $\Psi$ 's in the Lagrangian (4.12) are organized into two chiral and two Fermi multiplets, all sitting in the adjoint representation. The gauge field is promoted to a $\mathrm{U}(N)$ vector multiplet $\left(A_{\mu}, \lambda_{+}, \bar{\lambda}_{+}, D\right)$, whereas the scalar $\sigma$ is promoted to an adjoint chiral multiplet $\left(Y, \bar{\lambda}_{-}\right)$with the lowest component $Y=\rho+i \sigma$. The off-shell $\mathcal{N}=(0,2)$ supersymmetric version of the topological Yang-Mills Lagrangian is

$$
\mathcal{L}_{\text {top }}=\frac{i k}{2 \pi} \operatorname{Tr}\left(\sigma F_{12}-\rho D+\lambda_{+} \bar{\lambda}_{-}+\lambda_{-} \bar{\lambda}_{+}\right) .
$$

The fields and their quantum numbers under the global symmetry $\mathrm{SU}(2)^{4}$ are summarized in the table 3 .

Since the gaugino $\lambda_{+}$in this model is an auxiliary field, we can try the SUSY path integral using the original Lagrangian $\mathcal{L}_{\text {top }}$, not the usual kinetic Lagrangians (5.4), for the path integral weight for the multiplets containing $A_{\mu}$ and $\sigma$. The path integral of these multiplets is then trivial, and we are left with an integral over the moduli of flat $\mathrm{U}(N)$ gauge fields and the 1-loop determinant arising only from the fields $Z, \tilde{Z}, \Psi_{ \pm}$and $\tilde{\Psi}_{ \pm}$. The elliptic genus would then be given by

$$
Z_{T^{2}} \sim \int \prod_{i=1}^{N} \frac{\mathrm{d} w_{i} \mathrm{~d} \bar{w}_{i}}{\operatorname{Im} \tau} \cdot \prod_{i, j} \frac{\theta_{1}\left(w_{i}-w_{j}+m+\frac{1}{2}\left(\epsilon_{1}+\epsilon_{2}\right)\right) \theta_{1}\left(w_{i}-w_{j}+m-\frac{1}{2}\left(\epsilon_{1}+\epsilon_{2}\right)\right)}{\theta_{1}\left(w_{i}-w_{j}+\epsilon_{1}\right) \theta_{1}\left(w_{i}-w_{j}+\epsilon_{2}\right)} .
$$


However, there is a priori no natural way to rewrite it further into some integral of an $(N, 0)$-form. Similar formulae for the elliptic genus have been proposed for theories with Stückelberg fields in $[37,38]$, though in those cases the elliptic genus becomes a non holomorphic function of $\tau$.

The integral with respect to $w_{i}, \bar{w}_{i}$ is finite and does not require careful regularization. For $N=1$ the integrand does not depend on $w_{1}, \bar{w}_{1}$ at all, so the above $Z_{T^{2}}$ agrees with the result of IIA brane model (5.23). For higher $N$, the integrand contains the $N$-power of the U(1) part (5.23), so it vanishes faster than the elliptic genus of the IIA brane model as $m \rightarrow \frac{1}{2}\left(\epsilon_{1}+\epsilon_{2}\right)$. This is due to the Fermi multiplet $\tilde{\Psi}_{+}$which acquire $N$ zeromodes in this limit. Thus the elliptic genera of the IIA brane model and that of ABJM slab do not agree.

When $2 m=\epsilon_{1}+\epsilon_{2}$, then the Fermi multiplet $\tilde{\Psi}_{+}$couples to no external gauge fields, so one can consider turning off $\mathcal{L}_{\text {top }}$ in the original Lagrangian and instead add standard kinetic term $\mathcal{L}_{\text {vec }}$ for the vector multiplet, and also a mass term which is bilinear in the multiplets $Y$ and $\tilde{\Psi}_{+}$. After neglecting the fields which become massive, the remaining field content is the same as that of $\mathcal{N}=(4,4) \mathrm{U}(N)$ super Yang-Mills theory. This deformation should correspond to turning on $u_{A}$ and integrating out the scalar $\sigma$ in section 4.1. Although the deformation is SUSY exact, it involves the multiplets with zeromodes and therefore changes the asymptotic behavior of path integral weight. Under such deformation the elliptic genus may well change. Indeed, while (5.26) gives rise to $N$ powers of zeroes at $m=\frac{1}{2}\left(\epsilon_{1}+\epsilon_{2}\right)$, the elliptic genus for $\mathrm{U}(N)$ super Yang-Mills theory has only one zero.

Young diagram sum from Jeffrey-Kirwan residue. After setting $m=\frac{1}{2}\left(\epsilon_{1}+\epsilon_{2}\right)$ and removing the $\mathrm{U}(1)$ part (which is vanishing due to a fermion zeromode), the elliptic genus of the remaining $\mathrm{SU}(N)$ super Yang-Mills theory is given by the Jeffrey-Kirwan residue of the following meromorphic form,

$$
Z_{1 \text {-loop }}=\prod_{i=1}^{N-1} \mathrm{~d} \hat{w}_{i} \cdot \frac{1}{N !}\left(\frac{\theta_{1}^{\prime} \theta_{1}\left(\epsilon_{1}+\epsilon_{2}\right)}{\theta_{1}\left(\epsilon_{1}\right) \theta_{1}\left(\epsilon_{2}\right)}\right)^{N-1} \prod_{i \neq j} \frac{\theta_{1}\left(w_{i}-w_{j}\right) \theta_{1}\left(w_{i}-w_{j}-\epsilon_{1}-\epsilon_{2}\right)}{\theta_{1}\left(w_{i}-w_{j}-\epsilon_{1}\right) \theta_{1}\left(w_{i}-w_{j}-\epsilon_{2}\right)}
$$

Here the coordinates $w_{i}$ are assumed to satisfy $\sum_{i} w_{i}=0$. More explicitly, they are expressed in terms of the moduli of flat $\mathrm{SU}(N)$ gauge fields $\hat{w}_{i}$ as follows,

$$
\left(w_{1}, \cdots, w_{N}\right)=\left(-\hat{w}_{1}, \hat{w}_{1}-\hat{w}_{2}, \hat{w}_{2}-\hat{w}_{3}, \cdots, \hat{w}_{N-2}-\hat{w}_{N-1}, \hat{w}_{N-1}\right)
$$

The coordinates $\hat{w}_{i}$ obey the periodicity $\hat{w}_{i} \sim \hat{w}_{i}+1 \sim \hat{w}_{i}+\tau$.

In the previous subsection we have shown that the elliptic genus of $\mathcal{N}=(4,4)$ super Yang-Mills theory is expressed as a sum over contributions labeled by Young diagrams, namely (5.22) with $m=\frac{1}{2}\left(\epsilon_{1}+\epsilon_{2}\right)$ substituted and the $\mathrm{U}(1)$ part removed. The derivation there was based on the Higgs branch localization in a system with fundamental matters. Here we wish to re-derive the same result as the Jeffrey-Kirwan residue of the meromorphic form (5.27).

The singular hyperplanes of $Z_{1 \text {-loop }}(5.27)$ are given by $w_{i}-w_{j}=\epsilon_{1}$ or $w_{i}-w_{j}=\epsilon_{2}$. It is convenient to describe their intersections graphically by arrangements of $N$ particles on a $2 \mathrm{D}$ square lattice. For example, some poles in the case $N=4$ and the corresponding 
graphs are

$$
\begin{aligned}
& \left.\left\{w_{2}-w_{1}=\epsilon_{1}, w_{3}-w_{2}=\epsilon_{1}, w_{4}-w_{3}=\epsilon_{1}\right\} \Longleftrightarrow \epsilon_{1} \Longleftrightarrow w_{3}-w_{1}=\epsilon_{2}, w_{4}-w_{2}=\epsilon_{1}\right\} \Longleftrightarrow w_{2}-w_{1}=\epsilon_{1}
\end{aligned}
$$

Note that the singular hyperplanes correspond to the links connecting particles occupying the neighboring sites. Some other poles, for example

$$
\begin{aligned}
\left\{w_{2}-w_{1}=\epsilon_{1},\right. & \left.w_{3}-w_{1}=\epsilon_{1}, w_{4}-w_{2}=\epsilon_{1}, w_{4}-w_{3}=\epsilon_{1}\right\} \Longleftrightarrow \\
\left\{w_{2}-w_{1}=\epsilon_{1}, w_{3}-w_{2}=\epsilon_{1}, w_{4}-w_{3}=\epsilon_{2}\right\} & \Longleftrightarrow
\end{aligned}
$$

will have vanishing residues due to the factors in the enumerator of $Z_{1 \text {-loop }}$. In the first example where $w_{2}$ and $w_{3}$ occupy the same lattice site, the residue vanishes due to the factor $\theta_{1}\left(w_{2}-w_{3}\right)^{2}$ although 4 hyperplanes are intersecting there. In the second example the residue vanishes due to the enumerator factor $\theta_{1}\left(w_{4}-w_{2}-\epsilon_{1}-\epsilon_{2}\right)$ which is indicated by the slash (thin oblique line) in the graph.

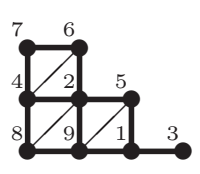

Let us first focus on the poles corresponding to Young tableaux (= Young diagrams with numbering of boxes). The figure on the right shows an example of a pole for the case $N=9$, where 11 hypersurfaces (corresponding to 5 vertical and 6 horizontal links) intersect, and 3 zeroes arising from the enumerator. For a pole corresponding to a general Young tableau $\mathbf{T}$, we consider the simplified meromorphic form,

$$
\hat{Z}_{1 \text {-loop }}(\mathbf{T})=\mathrm{d}^{N-1} \hat{w} \cdot \frac{\prod_{\nearrow}\left(w_{j}-w_{i}-\epsilon_{1}-\epsilon_{2}\right)}{\prod_{\rightarrow}\left(w_{j}-w_{i}-\epsilon_{1}\right) \cdot \prod_{\uparrow}\left(w_{j}-w_{i}-\epsilon_{2}\right)},
$$

which extracts the singular and vanishing factors from $Z_{1 \text {-loop }}$ in (5.27). The three products are over the slashes, horizontal links and vertical links, respectively. Note that $\hat{Z}_{1 \text {-loop }}$ has degree $N-1$ for any tableau $\mathbf{T}$ of $N$ boxes, namely $\hat{Z}_{1 \text {-loop }}$ has always $N-1$ more factors in the denominator than in the enumerator.

We use the following identities

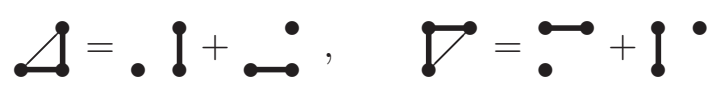

to rewrite $\hat{Z}_{1 \text {-loop }}(\mathbf{T})$ into a sum of basic fractions. Here is an $N=6$ example (the numbering of particles is suppressed).

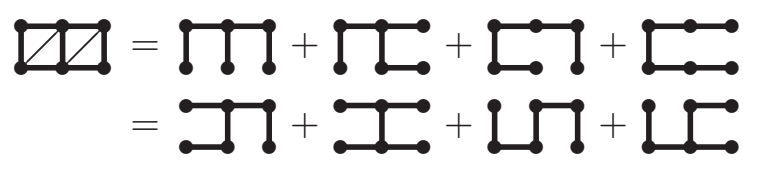


Each basic fraction is described by a tree graph of $N-1$ links connecting $N$ particles. Note that the decomposition of $\hat{Z}_{\text {1-loop }}(\mathbf{T})$ into basic fractions is in general not unique. Once the numbering of $N$ particles is restored, one can associate to each basic fraction a set $b$ of $N-1$ charge vectors $(\mathrm{SU}(N)$ roots). For example we have,

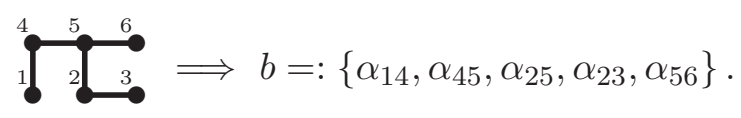

Here we identified the $\mathrm{SU}(N)$ root $\alpha_{i j}$ with the $N$-component vector whose $i$-th component is $-1, j$-th component +1 and others zero, so that $\alpha_{i j} \cdot w=w_{j}-w_{i}$.

To evaluate the Jeffrey-Kirwan residue, we choose $\eta$ to be a generic $N$-component vector satisfying

$$
\eta_{1}=-\left(\eta_{2}+\cdots+\eta_{N}\right), \quad \eta_{2}>0, \cdots, \eta_{N}>0
$$

For each tree graph such as (5.34), we need to determine whether $\eta$ is inside the cone generated by the charge vectors in $b$. To do this graphically, we think of a current flowing along the tree graph. The lattice site occupied by the $i$-th particle $(i \neq 1)$ is a source of strength $\eta_{i}$, and the site occupied by the 1-st particle is a sink of strength $\left(\eta_{2}+\cdots+\eta_{N}\right)$. We then compute the flow along each link. For example, for the graph given in (5.34) we find

$$
\begin{aligned}
& \underset{\sim}{\longrightarrow \sim \eta_{3}} \Longrightarrow \eta=\left(\eta_{2}+\eta_{3}+\eta_{4}+\eta_{5}+\eta_{6}\right) \alpha_{14}+\left(\eta_{2}+\eta_{3}+\eta_{5}+\eta_{6}\right) \alpha_{45} \\
& \begin{array}{c}
\eta_{2} \\
\eta_{2}+\eta_{3}+\eta_{4}+\eta_{5}+\eta_{6}
\end{array} \\
& -\left(\eta_{2}+\eta_{3}\right) \alpha_{25}+\eta_{3} \alpha_{23}+\eta_{6} \alpha_{56} \text {. }
\end{aligned}
$$

This shows $\eta$ is outside of the cone generated by $b$ of (5.34) because the coefficient of $\alpha_{25}$ is negative.

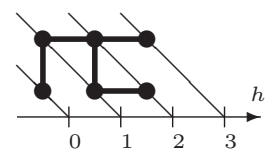

In order to make more general statements, it is useful to introduce the height function $h$ on tree graphs. As shown in the figure on the right, we assign height 0 to the unique bottom-left corner site of the graph. The height increases (decreases) by one as we move along the tree graph one step up or right (down or left). Looking back at the computation (5.36), we notice that the minus sign in a coefficient corresponds to a backward flow of current along the link between 2 and 5 . For $\eta$ to be inside the cone, the tree graph and the arrangement of $N$ particles on it should have been such that the current be flowing everywhere in accordance with the height gradient.

For a basic fraction to have nonzero Jeffrey-Kirwan residue, the corresponding tree graph has to be monotonic, which means the following. The 1-st particle is at the lowest site, and the height $h$ increases as one goes away from the 1-st particle along any path on the tree graph until one reaches an end. For the $\hat{Z}_{1 \text {-loop }}(\mathbf{T})$ to have nonzero JeffreyKirwan residue, its decomposition into basic fractions must contain a term corresponding to monotonic tree graph, therefore the number 1 must be assigned to the bottom-left corner of $\mathbf{T}$. In the sample decomposition of $\hat{Z}_{1 \text {-loop }}$ into basic fractions (5.33), we notice that in 
each line there is only one monotonic tree graph, namely the last term in each line. One can actually show that the decomposition always gives rise to only one monotonic tree graph irrespective of $\mathbf{T}$ or the ways of decomposition, as follows. The only monotonic tree graph can be obtained by choosing, when applying the identities (5.32), always the second term on the r.h.s. An important characteristic of monotonic tree graph is that, if it has $N_{h}$ points at height $h$, it has exactly $N_{h}$ links between points of heights $h-1$ and $h$. This property is violated once one makes the other choice when applying (5.32).

The Jeffrey-Kirwan residue of a basic fraction corresponding to a tree diagram is, if nonzero, always given by the inverse of the determinant of $\mathrm{SU}(N)$ Cartan matrix.

$$
\operatorname{JK}-\operatorname{Res}(\eta)\left[\frac{\mathrm{d}^{N-1} \hat{w}}{\prod_{\alpha \in b} \alpha \cdot w}\right]= \begin{cases}N^{-1} & \text { if } \eta \in \operatorname{Cone}(b) \\ 0 & \text { otherwise. }\end{cases}
$$

Thus we have, for any Young tableau T,

$$
\operatorname{JK-Res}(\eta) \hat{Z}_{1-\text { loop }}(\mathbf{T})= \begin{cases}N^{-1} & \text { if } 1 \text { is in the bottom-left corner of } \mathbf{T} \\ 0 & \text { otherwise. }\end{cases}
$$

Note also that, for each Young diagram of $N$ boxes there are $(N-1)$ ! Young tableaux with the number 1 occupying the bottom left corner. There is yet another factor of $N^{2}$ arising from the fact that there are $N^{2}$ poles in the moduli space of flat $\mathrm{SU}(N)$ gauge fields corresponding to the same Young tableau. Namely, if there is a pole corresponding to a Young tableau at $w_{i}=w_{i}^{\circ}(i=1, \cdots, N)$, then there are actually $N^{2}$ poles corresponding to the same tableau at

$$
w_{i}=w_{i}^{\circ}+\frac{k+l \tau}{N} \cdot(k, l \in\{0,1, \cdots, N-1\})
$$

The product of these factors $N^{-1} \cdot(N-1) ! \cdot N^{2}$ precisely cancels with the order of Weyl group in the denominator of (5.27). Thus one can show, assuming that other poles not corresponding to any Young tableau do not contribute, that the Jeffrey-Kirwan residue of $Z_{1-\text { loop }}$ (5.27) reproduces the Young diagram sum (5.22) with $2 m=\epsilon_{1}+\epsilon_{2}$ substituted.

Let us now think of more general arrangements $\mathbf{A}$ of $N$ particles on a lattice, not necessarily corresponding to Young tableaux. Consider the Jeffrey-Kirwan residue of the following meromorphic form

$$
\hat{Z}_{\text {1-loop }}(\mathbf{A})=\mathrm{d}^{N-1} w \cdot \frac{\prod_{(\cdot)}\left(w_{j}-w_{i}\right)^{2} \prod_{\nearrow}\left(w_{j}-w_{i}-\epsilon_{1}-\epsilon_{2}\right)}{\prod_{\rightarrow}\left(w_{j}-w_{i}-\epsilon_{1}\right) \cdot \prod_{\uparrow}\left(w_{j}-w_{i}-\epsilon_{2}\right)},
$$

which is the simplified version of $Z_{1 \text {-loop }}$ at the pole corresponding to $\mathbf{A}$. The first factor in the enumerator is the product of double zeroes for pairs of particles occupying the same lattice site.

We apply to this meromorphic form the general formula for Jeffrey-Kirwan residue given at the beginning of this section. First, $\Delta$ in this case is the set of all the links 
connecting neighboring particles. Then the bases of $\Delta$ are identified with tree graphs, though now they are allowed to have overlapping particles or links. For arbitrary choice of the set $B$ of bases of $\Delta$, one should be able to write $\hat{Z}_{1 \text {-loop }}(\mathbf{A})$ as a sum of derivatives of basic fractions $\phi_{b}(b \in B)$ and fractions with trivial residue. The Jeffrey-Kirwan residue of the basic fraction $\phi_{b}$ is nonvanishing only when the corresponding tree graph is monotonic. In order for $\Delta$ to have at least one basis corresponding to a monotonic tree graph, the corresponding particle arrangement $\mathbf{A}$ must fit within the first quadrant and the 1-st particle has to sit at its bottom-left corner. Some simple examples of such A are those given in (5.29) and (5.30). Let us restrict our argument to such arrangements in what follows.

We arrange the elements of $\Delta$ so that the height of the corresponding links are nondecreasing. (Of course, this requirement does not fix the order uniquely.) Then, with a suitable choice of the basis set $B$, one can express the Jeffrey-Kirwan residue as a linear sum of iterated residues $\operatorname{Res}_{b}$, where the sum runs only over those $b \in B$ corresponding to monotonic tree graphs. In all of these iterated residues, one first uses the translation invariance of $\hat{Z}_{1 \text {-loop }}$ to fix $w_{1}$ corresponding to the 1-st particle at the corner site of height 0 , then integrate the $w_{i}$ 's corresponding to the particles at height 1 , then those corresponding to the height 2 and so on. This procedure can be viewed as putting one box after another in the first quadrant. If at each step of iteration a box is put in an incorrect place that violates the rule of making a Young diagram, the residue of $\hat{Z}_{1 \text {-loop }}(\mathbf{A})$ vanishes due to the effect of its enumerator. Thus the only contribution to the elliptic genus is from the poles corresponding to Young tableaux with the number 1 at the bottom left corner.

\section{Concluding remarks}

Our analysis of the ABJM model with boundary was able to reproduce the known observables for multiple self-dual strings only partially, and there remains a significant mismatch. We believe that it should be resolved by a deeper understanding of the ABJM model on the boundary and the IIA brane model.

In making more precise comparisons between the two descriptions through elliptic genera or other $R$-independent physical observables, one also needs to be careful about what those observables really are. A good example where such a subtlety arises is a microscopic derivation of the quarter-BPS-dyon counting formula in $\mathcal{N}=4$ string theory using the $4 \mathrm{~d}-5 \mathrm{~d}$ lift [40]. We start with the D1-D5 system in IIB on $K 3 \times S^{1} \times$ Taub-NUT. When the radius $R$ of the asymptotic circle of the Taub-NUT space is large, the above system describes the Strominger-Vafa black hole with angular momentum. When $R$ is small, the system can be related to a quarter BPS dyon using the string duality. Thus one can expect that the partition function of these dyons, independent of $R$, equals to that of the rotating Strominger-Vafa black holes. The former is given by the so-called Igusa form and the latter by the elliptic genus of symmetric products of $K 3$. Indeed they are very similar but not exactly the same. To have the exact match, it turns out that one also needs to consider other contributions such as the center of mass motion of the D1-D5 system in the Taub-NUT space and bound states of momentum along $S^{1}$ with the KK monopole [41]. 
The above example suggests that, in order to have an exact match between the elliptic genera of the ABJM slab and the IIA brane model, we may also need to take care of those additional contribution such as the c.o.m motion of the M2-M5 system in the Taub-NUT space. We leave this for the future.

In the present work, we studied the simplest boundary condition relevant to the worldsheet theory of the self-dual string, and did not consider possible boundary degrees of freedom such as those proposed in [9]. It would be very interesting to fully explore the $1 / 2$ BPS supersymmetric boundary conditions of the ABJM model along the line of [8]. In addition it is important to generalize our analysis to a system of M2-branes intersecting with M5-branes, i.e., domain wall to construct the quiver models discussed in section 4.4.

Another direction to study further is a generalization to $\mathcal{N}=5$ Chern-Simons-Matter theories $[42,43]$ on the boundary and interval, and other boundary conditions such as from the 9-branes [44].

\section{Acknowledgments}

We would like to thank Kentaro Hori, Seok Kim, David Kutasov, Emil Martinec, Hirosi Ooguri and Soo-Jong Rey for discussions. We thank the organizers of the Simons Summer Workshop 2013 and the KITP program "New Methods in Nonperturbative Quantum Field Theory" where part of this work has been carried out. KH also thanks the organizers of the Kavli IPMU-FMSP Workshop "Supersymmetry in Physics and Mathematics" for hospitality. The work of KH is supported in part by MEXT/JSPS Grant-in-Aid for Scientific Research No. 26400247. The work of SL is supported in part by the Ernest Rutherford fellowship of the Science \& Technology Facilities Council ST/J003549/1.

\section{A $\quad \mathrm{U}(1) \times \mathrm{U}(1)$ ABJM model on a slab}

In order to study SUSY-protected sector of the worldsheet theory of self-dual strings, it may be essential to formulate the ABJM model on a slab in an off-shell supersymmetric manner including Kaluza-Klein degree of freedom. We need to reorganize the fields into multiplets of 2D SUSY, and regard each field as carrying an additional continuously-varying label $y$ in a way similar to $[8,39]$. Here we illustrate this procedure for the simplest example of Euclidean $\mathrm{U}(1) \times \mathrm{U}(1)$ ABJM theory. The Lagrangian on flat $\mathbb{R}^{3}$ is

$$
\begin{aligned}
\mathcal{L} & =\frac{i k}{4 \pi} \varepsilon^{m n p}\left(A_{m} \partial_{n} A_{p}-\tilde{A}_{m} \partial_{n} \tilde{A}_{p}\right)+D_{m} \bar{Z}^{a} D^{m} Z_{a}-\bar{\Psi}_{a} \gamma^{m} D_{m} \Psi^{a} \\
& =\frac{i k}{2 \pi} \varepsilon^{m n p} B_{m} \partial_{n} C_{p}+D_{m} \bar{Z}^{a} D^{m} Z_{a}-\bar{\Psi}_{a} \gamma^{m} D_{m} \Psi^{a}
\end{aligned}
$$

where $B_{m} \equiv \frac{1}{2}\left(A_{m}+\tilde{A}_{m}\right), C_{m} \equiv A_{m}-\tilde{A}_{m}$ and the covariant derivative of matters is $D_{m} Z_{a}=\left(\partial_{m}-i C_{m}\right) Z_{a}$ etc. We would like to put it on $S^{2} \times$ (interval) preserving 2D $\mathcal{N}=(2,2)$ off-shell supersymmetry associated with the Killing spinor on the round $S^{2}$ of radius $\ell$,

$$
D_{\mu} \epsilon=\frac{i}{2 \ell} \gamma_{\mu} \epsilon
$$


We use the same convention for the spinor calculus as was summarized at the beginning of section 3, except $\gamma^{m}(m=1,2,3)$ here are chosen to be Pauli's matrices. We follow the general construction of $\mathcal{N}=(2,2)$ theories on $S^{2}$ given in $[28,29]$.

The boundary conditions on various fields are

$$
\begin{array}{rlll}
\text { Neumann: } & B_{\mu}, & C_{3}, & Z_{I}, \Psi_{-}^{I}, \Psi_{+}^{A}, \\
\text { Dirichlet: } & C_{\mu}, & B_{3}, & Z_{A}, \Psi_{-}^{A}, \Psi_{+}^{I} . \quad(\mu=1,2 ; I=1,2 ; A=3,4)
\end{array}
$$

Note that the fields in the same multiplet must obey the same boundary condition.

Let us first focus on the terms in (A.1) involving the covariant $x_{\mu}$-derivative of matters. $C_{\mu}$ is in an abelian vector multiplet,

$$
\begin{aligned}
2 \delta C_{\mu} & =\xi \gamma_{\mu} \bar{\lambda}+\bar{\xi} \gamma_{\mu} \lambda, \\
2 \delta \sigma_{3} & =\xi \gamma_{3} \bar{\lambda}+\bar{\xi} \gamma_{3} \lambda, \\
2 \delta \sigma_{4} & =i \xi \bar{\lambda}-i \bar{\xi} \lambda \\
\delta \lambda & =i \gamma^{3} \xi F_{12}+\xi D+\gamma^{\mu 3} D_{\mu}\left(\xi \sigma_{3}\right)-i \gamma^{\mu} D_{\mu}\left(\xi \sigma_{4}\right), \\
\delta \bar{\lambda} & =i \gamma^{3} \bar{\xi} F_{12}-\bar{\xi} D+\gamma^{\mu 3} D_{\mu}\left(\bar{\xi} \sigma_{3}\right)+i \gamma^{\mu} D_{\mu}\left(\bar{\xi} \sigma_{4}\right), \\
2 \delta D & =D_{\mu}\left(\xi \gamma^{\mu} \bar{\lambda}\right)-D_{\mu}\left(\bar{\xi} \gamma^{\mu} \lambda\right),
\end{aligned}
$$

Here $F_{12}$ is the field strength of $C_{\mu}$. The matter fields are organized into four chiral multiplets with the lowest components $\phi_{i}(i=1, \cdots, 4)$, which couple to $C_{\mu}$ according to the charges $e_{i}=(+1,-1,+1,-1)$. Their transformation rule reads

$$
\begin{aligned}
\delta \phi_{i} & =\xi \psi_{i}, \\
\delta \bar{\phi}_{i} & =\bar{\xi} \bar{\psi}_{i} \\
\delta \psi_{i} & =-\gamma^{\mu} \bar{\xi} D_{\mu} \phi_{i}+i e_{i} \gamma^{3} \bar{\xi} \sigma_{3} \phi_{i}-e_{i} \bar{\xi} \sigma_{4} \phi_{i}+\xi F_{i}-\frac{q_{i}}{2} \gamma^{\mu} D_{\mu} \bar{\xi} \phi_{i}, \\
\delta \bar{\psi}_{i} & =-\gamma^{\mu} \xi D_{\mu} \bar{\phi}_{i}-i e_{i} \gamma^{3} \xi \sigma_{3} \bar{\phi}_{i}-e_{i} \xi \sigma_{4} \bar{\phi}_{i}-\bar{\xi}_{i}-\frac{q_{i}}{2} \gamma^{\mu} D_{\mu} \xi \bar{\phi}_{i}, \\
\delta F_{i} & =-\bar{\xi} \gamma^{\mu} D_{\mu} \psi_{i}+i e_{i} \bar{\xi} \gamma^{3} \sigma_{3} \psi_{i}+e_{i} \bar{\xi} \sigma_{4} \psi_{i}+i e_{i} \bar{\xi} \bar{\lambda} \phi_{i}-\frac{q_{i}}{2} D_{\mu} \bar{\xi} \gamma^{\mu} \psi_{i}, \\
\delta \bar{F}_{i} & =+\xi \gamma^{\mu} D_{\mu} \bar{\psi}_{i}+i e_{i} \xi \gamma^{3} \sigma_{3} \bar{\psi}_{i}-e_{i} \xi \sigma_{4} \bar{\psi}_{i}+i e_{i} \xi \lambda \bar{\phi}_{i}+\frac{q_{i}}{2} D_{\mu} \xi \gamma^{\mu} \bar{\psi}_{i} .
\end{aligned}
$$

Here $q_{i}$ are the vector R-charges. The component fields in these multiplets are identified with $Z_{a}, \Psi^{a}$ as follows,

$$
\begin{aligned}
\phi_{i} & =\left(Z_{1}, \bar{Z}^{2}, Z_{3}, \bar{Z}^{4}\right), \\
\psi_{i+} & =\left(\Psi_{+}^{3},-\bar{\Psi}_{4+},-\Psi_{+}^{1}, \bar{\Psi}_{2+}\right), \\
\psi_{i-} & =\left(\Psi_{-}^{2},-\bar{\Psi}_{1-}, \Psi_{-}^{4},-\bar{\Psi}_{3-}\right) .
\end{aligned}
$$

The above transformation rules are the same as the one for $2 \mathrm{D} \mathcal{N}=(2,2)$ SUSY theories on $S^{2}$, except that all the fields now depend also on $x^{3}$. The standard 2D SUSY construction gives a part of the matter kinetic term,

$$
\mathcal{L}_{\text {kin }}=D_{\mu} \bar{\phi}_{i} D^{\mu} \phi_{i}+\bar{\phi}_{i} e_{i}^{2}\left(\sigma_{3}^{2}+\sigma_{4}^{2}\right) \phi_{i}-\bar{\psi}_{i}\left(\gamma^{\mu} D_{\mu}-i e_{i} \gamma^{3} \sigma_{3}-e_{i} \sigma_{4}\right) \psi_{i}+\bar{F}_{i} F_{i}
$$




$$
+e_{i}\left(i \bar{\phi}_{i} D \phi_{i}-i \bar{\phi}_{i} \lambda \psi_{i}+i \bar{\psi}_{i} \bar{\lambda} \phi_{i}+\frac{i q_{i}}{\ell} \bar{\phi}_{i} \sigma_{4} \phi_{i}\right)+\frac{i q_{i}}{2 \ell} \bar{\psi}_{i} \psi_{i}+\frac{q_{i}\left(2-q_{i}\right)}{4 \ell^{2}} \bar{\phi}_{i} \phi_{i}
$$

where the summation over $i$ is understood.

The other part of the kinetic term, which involves covariant $x_{3}$-derivative of matter fields, should arise from F-term. We first introduce the chiral multiplet containing $C_{3}$,

$$
\begin{aligned}
\delta\left(C_{3}+i C_{4}\right) & =\xi \zeta \\
\delta\left(C_{3}-i C_{4}\right) & =\bar{\xi} \bar{\zeta} \\
\delta \zeta & =\gamma^{\mu} \bar{\xi}\left(\partial_{3} C_{\mu}-\partial_{\mu}\left(C_{3}+i C_{4}\right)\right)+\gamma^{3} \bar{\xi} \partial_{3} \sigma_{3}+i \bar{\xi} \partial_{3} \sigma_{4}+\xi H, \\
\delta \bar{\zeta} & =\gamma^{\mu} \xi\left(\partial_{3} C_{\mu}-\partial_{\mu}\left(C_{3}-i C_{4}\right)\right)+\gamma^{3} \xi \partial_{3} \sigma_{3}-i \xi \partial_{3} \sigma_{4}+\bar{\xi} \bar{H}, \\
\delta H & =-\bar{\xi} \gamma^{\mu} D_{\mu} \zeta+\bar{\xi} \partial_{3} \bar{\lambda} \\
\delta \bar{H} & =+\xi \gamma^{\mu} D_{\mu} \bar{\zeta}-\xi \partial_{3} \lambda .
\end{aligned}
$$

The above transformation rule is that of $\partial_{3} \cdot \log$ of an ordinary charged chiral multiplet, which ensures that $C_{3}$ transforms as the third component of the gauge field $C$ under gauge transformations. From the gauge-invariant superpotential,

$$
W=\phi_{2}\left(\partial_{3}-i C_{3}+C_{4}\right) \phi_{3}-\phi_{4}\left(\partial_{3}-i C_{3}+C_{4}\right) \phi_{1},
$$

we obtain the following F-term invariant

$$
\begin{aligned}
\mathcal{L}_{\text {F-term }}= & i H\left(\phi_{1} \phi_{4}-\phi_{2} \phi_{3}\right)+i \bar{H}\left(\bar{\phi}_{1} \bar{\phi}_{4}-\bar{\phi}_{2} \bar{\phi}_{3}\right) \\
& -F_{4}\left(D_{3} \phi_{1}+C_{4} \phi_{1}\right)-F_{3}\left(D_{3} \phi_{2}-C_{4} \phi_{2}\right)+F_{2}\left(D_{3} \phi_{3}+C_{4} \phi_{3}\right)+F_{1}\left(D_{3} \phi_{4}-C_{4} \phi_{4}\right) \\
& +\bar{F}_{4}\left(D_{3} \bar{\phi}_{1}+C_{4} \bar{\phi}_{1}\right)+\bar{F}_{3}\left(D_{3} \bar{\phi}_{2}-C_{4} \bar{\phi}_{2}\right)-\bar{F}_{2}\left(D_{3} \bar{\phi}_{3}+C_{4} \bar{\phi}_{3}\right)-\bar{F}_{1}\left(D_{3} \bar{\phi}_{4}-C_{4} \bar{\phi}_{4}\right) \\
& +\psi_{4}\left(D_{3}+C_{4}\right) \psi_{1}-\psi_{2}\left(D_{3}+C_{4}\right) \psi_{3}+\bar{\psi}_{4}\left(D_{3}+C_{4}\right) \bar{\psi}_{1}-\bar{\psi}_{2}\left(D_{3}+C_{4}\right) \bar{\psi}_{3} \\
& -i \zeta\left(\phi_{4} \psi_{1}+\psi_{4} \phi_{1}-\phi_{2} \psi_{3}-\psi_{2} \phi_{3}\right)+i \bar{\zeta}\left(\bar{\phi}_{4} \bar{\psi}_{1}+\bar{\psi}_{4} \bar{\phi}_{1}-\bar{\phi}_{2} \bar{\psi}_{3}-\bar{\psi}_{2} \bar{\phi}_{3}\right) .
\end{aligned}
$$

Note that, due to the superpotential, the R-charges of matter chiral multiplet must satisfy $q_{1}+q_{4}=q_{2}+q_{3}=2$. In addition, if we are putting nonzero classical values to $\phi_{3}=Z_{3}$ and $\phi_{4}=\bar{Z}^{4}$, the supersymmetry will be broken unless $q_{3}=q_{4}=0$.

Let us introduce another gauge field $B_{m}$ and construct Chern-Simons Lagrangian. The first two components $B_{\mu}$ belong to a chiral multiplet of vector R-charge $q=2$ or "twisted vector multiplet,

$$
\begin{aligned}
2 \delta B_{\mu} & =\xi \gamma_{\mu 3} \bar{\chi}+\bar{\xi} \gamma_{\mu 3} \chi \\
\delta\left(\rho_{3}+i \rho_{4}\right) & =\xi \chi \\
\delta\left(\rho_{3}-i \rho_{4}\right) & =\bar{\xi} \bar{\chi} \\
\delta \chi & =-i \gamma^{\mu} D_{\mu}\left(\bar{\xi}\left(\rho_{3}+i \rho_{4}\right)\right)+\xi\left(E+i G_{12}\right), \\
\delta \bar{\chi} & =-i \gamma^{\mu} D_{\mu}\left(\xi\left(\rho_{3}-i \rho_{4}\right)\right)-\bar{\xi}\left(E-i G_{12}\right), \\
\delta\left(E+i G_{12}\right) & =-D_{\mu}\left(\bar{\xi} \gamma^{\mu} \chi\right), \\
\delta\left(E-i G_{12}\right) & =+D_{\mu}\left(\xi \gamma^{\mu} \bar{\chi}\right),
\end{aligned}
$$


where $G_{12}$ is the field strength of $B_{\mu}$. $B_{3}$ belongs to a twisted chiral multiplet of axial R-charge 0 which couples to the above twisted vector multiplet in the same way the chiral multiplet $C_{3}$ couples to the vector multiplet $C_{\mu}$.

$$
\begin{aligned}
2 \delta B_{3} & =\xi \gamma_{3} \bar{\eta}+\bar{\xi} \gamma_{3} \eta \\
2 \delta B_{4} & =i \xi \bar{\eta}-i \bar{\xi} \eta \\
\delta \eta & =i \gamma^{3} \xi K_{4}+\xi K_{3}+\gamma^{\mu 3} \xi D_{\mu} B_{3}-i \gamma^{\mu} \xi D_{\mu} B_{4}+\bar{\xi} \partial_{3}\left(\rho_{3}+i \rho_{4}\right)-\gamma^{\mu 3} \xi \partial_{3} B_{\mu}, \\
\delta \bar{\eta} & =i \gamma^{3} \bar{\xi} K_{4}-\bar{\xi} K_{3}+\gamma^{\mu 3} \bar{\xi} D_{\mu} B_{3}+i \gamma^{\mu} \bar{\xi} D_{\mu} B_{4}+\xi \partial_{3}\left(\rho_{3}-i \rho_{4}\right)-\gamma^{\mu 3} \bar{\xi} \partial_{3} B_{\mu}, \\
2 \delta K_{3} & =\xi \gamma^{\mu} D_{\mu} \bar{\eta}-\bar{\xi} \gamma^{\mu} D_{\mu} \eta-\bar{\xi} \partial_{3} \chi+\xi \partial_{3} \bar{\chi} \\
2 \delta K_{4} & =i \xi \gamma^{\mu 3} D_{\mu} \bar{\eta}+i \bar{\xi} \gamma^{\mu 3} D_{\mu} \eta .+\bar{\xi} \gamma_{3} \partial_{3} \chi+\xi \gamma_{3} \partial_{3} \bar{\chi} .
\end{aligned}
$$

If we temporarily forget about the gauge non-invariance of $B_{3}, C_{3}$ and apply the standard construction of 2D F-term and twisted F-term, we obtain the following Chern-Simons like Lagrangian,

$$
\begin{aligned}
\mathcal{L}_{\mathrm{cs}}= & \frac{i k}{2 \pi}\left(F_{12} B_{3}+D B_{4}+K_{4} \sigma_{3}+K_{3} \sigma_{4}+G_{12} C_{3}+E C_{4}+H_{4} \rho_{3}+H_{3} \rho_{4}\right) \\
& +\frac{k}{4 \pi}(\lambda \bar{\eta}+\bar{\lambda} \eta-\chi \zeta-\bar{\chi} \bar{\zeta})
\end{aligned}
$$

where we used $H=H_{3}+i H_{4}, \bar{H}=H_{3}-i H_{4}$. This $\mathcal{L}_{\mathrm{cs}}$ is actually not supersymmetric, but it is cured by adding

$$
\delta \mathcal{L}_{\mathrm{cs}}=\frac{i k}{2 \pi}\left(B_{2} \partial_{3} C_{1}-B_{1} \partial_{3} C_{2}\right),
$$

which is also the right term to complete the Chern-Simons Lagrangian.

Open Access. This article is distributed under the terms of the Creative Commons Attribution License (CC-BY 4.0), which permits any use, distribution and reproduction in any medium, provided the original author(s) and source are credited.

\section{References}

[1] B. Haghighat, A. Iqbal, C. Kozcaz, G. Lockhart and C. Vafa, M-strings, arXiv:1305.6322 [INSPIRE].

[2] B. Haghighat, C. Kozcaz, G. Lockhart and C. Vafa, Orbifolds of M-strings, Phys. Rev. D 89 (2014) 046003 [arXiv:1310.1185] [InSPIRE].

[3] O. Aharony, O. Bergman, D.L. Jafferis and J. Maldacena, $N=6$ superconformal Chern-Simons-matter theories, M2-branes and their gravity duals, JHEP 10 (2008) 091 [arXiv:0806.1218] [INSPIRE].

[4] D.S. Berman, M.J. Perry, E. Sezgin and D.C. Thompson, Boundary conditions for interacting membranes, JHEP 04 (2010) 025 [arXiv:0912.3504] [INSPIRE].

[5] M. Aganagic, H. Ooguri, N. Saulina and C. Vafa, Black holes, q-deformed $2 d$ Yang-Mills and non-perturbative topological strings, Nucl. Phys. B 715 (2005) 304 [hep-th/0411280] [INSPIRE]. 
[6] F. Benini, R. Eager, K. Hori and Y. Tachikawa, Elliptic genera of two-dimensional $N=2$ gauge theories with rank-one gauge groups, Lett. Math. Phys. 104 (2014) 465 [arXiv: 1305.0533] [INSPIRE].

[7] F. Benini, R. Eager, K. Hori and Y. Tachikawa, Elliptic genera of $2 d N=2$ gauge theories, arXiv: 1308.4896 [INSPIRE].

[8] D. Gaiotto and E. Witten, Supersymmetric boundary conditions in $N=4$ super Yang-Mills theory, J. Statist. Phys. 135 (2009) 789 [arXiv:0804.2902] [INSPIRE].

[9] C.-S. Chu and D.J. Smith, Multiple self-dual strings on M5-branes, JHEP 01 (2010) 001 [arXiv:0909.2333] [INSPIRE].

[10] W. Nahm, A simple formalism for the BPS monopole, Phys. Lett. B 90 (1980) 413 [INSPIRE].

[11] D.-E. Diaconescu, D-branes, monopoles and Nahm equations, Nucl. Phys. B 503 (1997) 220 [hep-th/9608163] [INSPIRE].

[12] A. Basu and J.A. Harvey, The M2-M5 brane system and a generalized Nahm's equation, Nucl. Phys. B 713 (2005) 136 [hep-th/0412310] [INSPIRE].

[13] D. Nogradi, M2-branes stretching between M5-branes, JHEP 01 (2006) 010 [hep-th/0511091] [INSPIRE].

[14] S. Terashima, On M5-branes in $N=6$ membrane action, JHEP 08 (2008) 080 [arXiv:0807.0197] [INSPIRE].

[15] J. Gomis, D. Rodriguez-Gomez, M. Van Raamsdonk and H. Verlinde, A massive study of M2-brane proposals, JHEP 09 (2008) 113 [arXiv:0807.1074] [INSPIRE].

[16] K. Hanaki and H. Lin, M2-M5 systems in $N=6$ Chern-Simons theory, JHEP 09 (2008) 067 [arXiv:0807.2074] [INSPIRE].

[17] T. Nosaka and S. Terashima, M5-branes in ABJM theory and Nahm equation, Phys. Rev. D 86 (2012) 125027 [arXiv:1208.1108] [InSPIRE].

[18] K. Sakai and S. Terashima, Integrability of BPS equations in ABJM theory, JHEP 11 (2013) 002 [arXiv:1308.3583] [INSPIRE].

[19] E. Witten, On quantum gauge theories in two-dimensions, Commun. Math. Phys. 141 (1991) 153 [INSPIRE].

[20] E. Witten, Two-dimensional gauge theories revisited, J. Geom. Phys. 9 (1992) 303 [hep-th/9204083] [INSPIRE].

[21] M. Blau and G. Thompson, Derivation of the Verlinde formula from Chern-Simons theory and the G/G model, Nucl. Phys. B 408 (1993) 345 [hep-th/9305010] [INSPIRE].

[22] M. Blau and G. Thompson, Chern-Simons theory on $S^{1}$-bundles: abelianisation and q-deformed Yang-Mills theory, JHEP 05 (2006) 003 [hep-th/0601068] [INSPIRE].

[23] M. Blau and G. Thompson, Lectures on $2 D$ gauge theories: topological aspects and path integral techniques, hep-th/9310144 [INSPIRE].

[24] M.R. Douglas and G.W. Moore, D-branes, quivers and ALE instantons, hep-th/9603167 [INSPIRE].

[25] A. Gadde and S. Gukov, $2 d$ index and surface operators, JHEP 03 (2014) 080 [arXiv: 1305.0266] [INSPIRE]. 
[26] A. Szenes and M. Vergne, Toric reduction and a conjecture of Batyrev and Materov, Invent. Math. 158 (2004) 453 [math/0306311].

[27] M. Brion and M. Vergne, Arrangement of hyperplanes. I. Rational functions and Jeffrey-Kirwan residue, Ann. Sci. École Norm. Sup. 32 (1999) 715 [math/9903178].

[28] F. Benini and S. Cremonesi, Partition functions of $N=(2,2)$ gauge theories on $S^{2}$ and vortices, arXiv:1206.2356 [INSPIRE].

[29] N. Doroud, J. Gomis, B. Le Floch and S. Lee, Exact results in $D=2$ supersymmetric gauge theories, JHEP 05 (2013) 093 [arXiv:1206.2606] [INSPIRE].

[30] H.-Y. Chen, H.-Y. Chen and J.-K. Ho, Connecting mirror symmetry in $3 d$ and $2 d$ via localization, arXiv:1312.2361 [INSPIRE].

[31] M. Fujitsuka, M. Honda and Y. Yoshida, Higgs branch localization of $3 d N=2$ theories, arXiv: 1312.3627 [INSPIRE].

[32] F. Benini and W. Peelaers, Higgs branch localization in three dimensions, JHEP 05 (2014) 030 [arXiv: 1312.6078] [INSPIRE].

[33] Y. Yoshida, Factorization of $4 d N=1$ superconformal index, arXiv:1403.0891 [INSPIRE].

[34] W. Peelaers, Higgs branch localization of $N=1$ theories on $S^{3} \times S^{1}$, JHEP 08 (2014) 060 [arXiv: 1403.2711] [INSPIRE].

[35] H.-C. Kim, S. Kim, E. Koh, K. Lee and S. Lee, On instantons as Kaluza-Klein modes of M5-branes, JHEP 12 (2011) 031 [arXiv:1110.2175] [INSPIRE].

[36] H. Nakajima and K. Yoshioka, Instanton counting on blowup. 1, Invent. Math. 162 (2005) 313 [math/0306198] [INSPIRE].

[37] S.K. Ashok, N. Doroud and J. Troost, Localization and real Jacobi forms, JHEP 04 (2014) 119 [arXiv:1311.1110] [INSPIRE].

[38] S. Murthy, A holomorphic anomaly in the elliptic genus, JHEP 06 (2014) 165 [arXiv: 1311.0918] [INSPIRE].

[39] D. Gaiotto and E. Witten, Janus configurations, Chern-Simons couplings, and the $\theta$-angle in $N=4$ super Yang-Mills theory, JHEP 06 (2010) 097 [arXiv:0804.2907] [INSPIRE].

[40] D. Gaiotto, A. Strominger and X. Yin, New connections between $4 D$ and $5 D$ black holes, JHEP 02 (2006) 024 [hep-th/0503217] [INSPIRE].

[41] J.R. David and A. Sen, CHL dyons and statistical entropy function from D1-D5 system, JHEP 11 (2006) 072 [hep-th/0605210] [rNSPIRE].

[42] K. Hosomichi, K.-M. Lee, S. Lee, S. Lee and J. Park, $N=5,6$ superconformal Chern-Simons theories and M2-branes on orbifolds, JHEP 09 (2008) 002 [arXiv:0806.4977] [INSPIRE].

[43] O. Aharony, O. Bergman and D.L. Jafferis, Fractional M2-branes, JHEP 11 (2008) 043 [arXiv: 0807.4924] [INSPIRE].

[44] B. Haghighat, G. Lockhart and C. Vafa, $E+E \rightarrow$ H, Phys. Rev. D 90 (2014) 126012 [arXiv: 1406.0850] [INSPIRE]. 